\title{
Scaling of the strength of the meridional overturning with vertical diffusivity in an idealized global geometry
}

\author{
By MATTHIJS DEN TOOM* and HENK A. DIJKSTRA, Institute for Marine and Atmospheric \\ Research Utrecht, Department of Physics and Astronomy, Utrecht University, Princetonplein 5, \\ 3584 CC Utrecht, the Netherlands
}

(Manuscript received 31 March 2010; in final form 22 November 2010)

\begin{abstract}
An important expression of the non-linear character of the ocean's meridional overturning circulation (MOC) is the scaling of its amplitude with the magnitude of the vertical mixing coefficient (diffusivity) of heat and salt. This paper extends recent work that indicated that the Atlantic and Pacific MOC exhibit different scaling behaviour. An idealized two-basin model configuration is used to study the meridional overturning circulation under restoring boundary conditions. In particular, the effects of wind forcing and the choice of the parametrization of lateral mixing are examined.

Without wind, the scaling is similar in the two basins and consistent with theoretical predictions, provided that the diffusivity is small enough. Towards higher diffusivities the scaling of the overturning diverges. With non-zero wind, the sensitivity is strongly determined by the choice of the lateral mixing scheme. In case of traditional horizontal diffusion, the scaling behaviour is asymmetric due to spurious diapycnal mixing. With the Gent-McWilliams parametrization, the scaling is symmetric for the lower range of diffusivities, where results agree partly with theoretical scaling relations. In all cases considered, the pycnocline depth has the same sensitivity to diffusivity in both basins, implying that there is no universally valid relation between overturning strength and pycnocline depth.
\end{abstract}

\section{Introduction}

The transport of heat and salt by ocean currents modifies the density of ocean water. Density variations, in turn, induce pressure gradients that affect the ocean currents. This interaction is responsible for the non-linear dynamics of the large-scale circulation. Due to the ocean's important role in shaping the climate, understanding its complex behaviour is crucial for predicting future changes.

In this work, we extend recent process-oriented studies (Dalan et al., 2005; Dijkstra, 2008) that show that the scaling of the strength of the meridional overturning circulation (MOC) with vertical (or diapycnal) diffusivity $\left(K_{\mathrm{V}}\right)$ is different between the Pacific and Atlantic Oceans. The question of the circulation's sensitivity to $K_{\mathrm{V}}$ is rooted in the now long standing thermocline problem (Welander, 1959; Robinson and Stommel, 1959; Welander, 1971). A complete theory for the observed structure of oceanic density profiles would greatly advance our understanding of the deep circulation and its sensitivity to external

\footnotetext{
*Corresponding author.

e-mail: M.denToom@uu.nl

DOI: $10.1111 /$ j.1600-0870.2010.00496.x
}

parameters. It is still not clear, however, to what extent the MOC is governed by adiabatic dynamics, or, phrased differently, how important irreversible mixing is for bringing deep water to the surface (Kuhlbrodt et al., 2007).

Unfortunately, the available observational data are not comprehensive enough to be conclusive. Assuming that, on a global scale, the density tendency due to upwelling (vertical advection) is balanced solely by vertical mixing, a vertical diffusivity of about $1 \mathrm{~cm}^{2} \mathrm{~s}^{-1}$ is inferred from density profiles (Munk, 1966). This number is about one order of magnitude larger than the value suggested by direct measurements in the main thermocline (Ledwell et al., 1993). Mixing rates, however, were shown to have a high degree of spatial variability, with estimates for $K_{\mathrm{V}}$ ranging from $0.1 \mathrm{~cm}^{2} \mathrm{~s}^{-1}$ for the interior ocean over smooth abyssal plains to larger than $10 \mathrm{~cm}^{2} \mathrm{~s}^{-1}$ over rough topography (Toole et al., 1994; Polzin et al., 1997). The 'canonical' diffusivity found by Munk (1966) may therefore be reinterpreted as a surrogate for a small number of concentrated source regions of intense mixing (Munk and Wunsch, 1998). Yet, as long as the sparsity of direct measurements precludes a direct test of this idea, studies addressing the scaling of the overturning with vertical diffusivity remain valuable.

Among the simplest three-dimensional models of the ocean are those that consider buoyancy-driven flows in an idealized 
North Atlantic basin. Results for this configuration have often been compared to the 'classical' scaling laws (Lineikin, 1955; Robinson and Stommel, 1959; Bryan and Cox, 1967). Those are obtained by assuming that the flow is in geostrophic and hydrostatic balance and that an upwelling-diffusion balance controls the depth of the thermocline. In case of restoring surface conditions and a linear equation of state there is effectively one tracer and the classical scaling relations are given by

$V \sim \frac{g \Delta \rho}{\rho_{0} f L} \delta_{\rho}, \quad W \sim \frac{K_{\mathrm{V}}}{\delta_{\rho}}, \quad \Psi \sim V \delta_{\rho} L \sim W L^{2}$,

$\delta_{\rho} \sim\left(\frac{\rho_{0} f L^{2}}{g}\right)^{1 / 3}\left(\frac{K_{\mathrm{V}}}{\Delta \rho}\right)^{1 / 3}$

$\Psi \sim\left(\frac{L^{4} g}{\rho_{0} f}\right)^{1 / 3}\left(K_{\mathrm{V}}^{2} \Delta \rho\right)^{1 / 3}$.

Here, $V(W)$ is the characteristic horizontal (vertical) velocity, $\Psi$ is the strength of the MOC and $\delta_{\rho}$ is a measure of the pycnocline depth. In addition to $K_{\mathrm{V}}$, the independent parameters are $g$, the acceleration due to gravity, $\Delta \rho$, the equator-to-pole density difference, $\rho_{0}$, a reference density, $f$, a characteristic value of the Coriolis parameter and $L$, a characteristic horizontal length scale. Park and Bryan (2000) established a good agreement between these relations and their model results, and were able to explain the deviations from classical scaling found in earlier studies (Bryan, 1987; Colin de Verdière, 1988; Hu, 1996; Marotzke, 1997).

In the presence of wind stress the depth scale of the main thermocline may be controlled by the Ekman pump velocity $W_{\mathrm{E}}$. As a result, the sensitivity of the overturning circulation reduces to an approximate $K_{\mathrm{V}}^{1 / 2}$-dependence for small vertical diffusivities (Vallis, 2000). The effect of wind may even be more profound when the basin is extended to include both hemispheres and a zonally periodic channel, as a model for the Antarctic Circumpolar Current. Because no net geostrophic transport is possible across the unblocked latitudes of Drake Passage, the wind driven surface flow may induce a large-scale deep return flow, even in the absence of vertical mixing ('Drake Passage effect'; Toggweiler and Samuels, 1998). Gnanadesikan (1999) constructed a simple model for the pycnocline that incorporates this process, as well as the partially counteracting effect of the return flow induced by mesoscale eddies. Several flow regimes predicted by the model of Gnanadesikan (1999) have been shown to occur, both in a $z$-coordinate and an isopycnal ocean model, for a double-hemispheric basin with a southern channel (Klinger et al., 2003).

As a final extension, (semi-) realistic multibasin geometries can be considered. The sensitivity of the overturning circulation to $K_{\mathrm{V}}$ was studied in an idealized two-basin configuration by Dalan et al. (2005), using a coupled ocean-atmosphere model. Their results show that the Atlantic $\operatorname{MOC}\left(\Psi_{A}\right)$ scales with $K_{\mathrm{V}}$ with a power of 0.44 , while the Southern Pacific MOC $\left(\Psi_{\mathrm{P}}\right)$ scales with a power of 0.63 . The power law exponent for the Pacific overturning is thus quite close to the classical value of $2 / 3$, but the exponent for $\Psi_{\mathrm{A}}$ is clearly smaller. More recently, Dijkstra (2008) analysed the scaling behaviour of the MOC in a fully implicit global ocean-only model. Using restoring conditions for salinity and a simple energy balance atmospheric model to control upper ocean temperatures, he found that $\Psi_{\mathrm{A}}$ scales as $K_{\mathrm{V}}^{1 / 3}$, whereas $\Psi_{\mathrm{P}}$ scales as $K_{\mathrm{V}}^{2 / 3}$.

Dijkstra (2008) proposed the following tentative explanation for this asymmetry in scaling. His results suggest that the depth of the Atlantic pycnocline $\left(\delta_{\mathrm{A}}\right)$ hardly changes with $K_{\mathrm{V}}$, because it is controlled by advection, while the depth of Pacific pycnocline $\left(\delta_{\mathrm{P}}\right)$ is determined by classical scaling. In addition, he argued that the connection of the Pacific and Atlantic basins through the Southern Ocean implies that there is a single characteristic horizontal velocity $(V)$ that applies to the entire domain. Provided that $V$ scales as $K_{\mathrm{V}}^{1 / 3}$, the relations $\Psi_{\mathrm{A}} \sim V \delta_{\mathrm{A}}$ and $\Psi_{\mathrm{P}} \sim V \delta_{\mathrm{P}}$ result in the observed MOC scaling.

A crucial element in this idea is that the surface boundary conditions allow variations in the Atlantic surface density. With reference to eq. (1), this means that $\Delta \rho$ cannot be considered an external parameter. The same implication holds for the suggestion of Dalan et al. (2005) that the difference in scaling is the result of their use of 'mixed' boundary conditions.

The main goal of the present work is to explore the scaling behaviour of a multibasin model for the contrasting case in which the surface density is (nearly) fixed, akin to the classical single-hemisphere experiments. Using a simplified model consisting of interconnected zonally averaged ocean basins, Wright and Stocker (1992) showed that the scaling can also be asymmetric when restoring boundary conditions apply for both temperature and salinity. Motivated by these results, we turn to the fully three-dimensional case and pose the question if the difference in scaling between the Pacific and Atlantic is a robust feature of the 'global' overturning that is not (solely) related to $\Delta \rho$ being part of the solution. We simplify the problem by using a highly idealized model geometry that ignores the contrast in size and latitudinal extent between the basins of the world ocean. Instead, we examine how the scaling behaviour in the Pacific and Atlantic is affected by (1) the presence of wind stress and (2) the parametrization of lateral mixing by mesoscale eddies.

As reviewed above, it has been shown for various configurations that the presence of wind stress alters the sensitivity of the flow to $K_{\mathrm{V}}$. Because the asymmetry in scaling has only been observed in models that include wind forcing (Wright and Stocker, 1992; Knutti et al., 2000; Dalan et al., 2005; Dijkstra, 2008), our comparison between cases with and without wind provides new insights in the problem. The $K_{\mathrm{V}}$-scaling may also be affected by the parametrization of lateral mixing, through the amount of (spurious) cross-isopycnal mixing implied by the scheme of 
choice. Yet, Knutti et al. (2000) concluded that the scaling behaviour of the zonally averaged model of Wright and Stocker (1992) is essentially unaffected when instead of horizontal mixing a more physical representation of mesoscale eddies (Gent and McWilliams, 1990) is used. Here, we evaluate if this conclusion still holds if the flow has zonal structure.

In contrast to most other studies, in which only a few quasisteady solutions are computed, the implicit model approach used by Dijkstra (2008) allows to determine scaling relations based on many exact steady solutions. Currently, an important limitation of this method is that it can only be used efficiently with horizontal tracer mixing. We therefore combine the implicit approach with traditional explicit time stepping and thus attain a more complete picture than would have been possible with each of the individual approaches.

This paper is organized as follows. Section 2 contains the model formulation and an introduction to the experiments performed for this study. Section 3 presents the scaling results, which we analyse in more detail in Section 4. The relevance of the results is discussed in Section 5 and the paper is concluded in Section 6.

\section{Model formulation and experiments}

In this section, we describe the idealized ocean model (Section 2.1) and the methodology used to determine the scaling behaviour (Section 2.2).

\subsection{The two-basin ocean model}

We use a highly idealized geometry (Fig. 1a), similar in design to that of several other process-oriented studies (Marotzke and Willebrand, 1991; Von der Heydt and Dijkstra, 2007). It consists of two interhemispheric basins connected in the south by a zonally periodic channel. As such it captures the elementary geometrical characteristics of the World Ocean. Both basins extend from $52^{\circ} \mathrm{S}$ to $68^{\circ} \mathrm{N}$ and are $60^{\circ}$ wide, and we hereafter refer to the left (right) basin as 'Pacific' ('Atlantic') Ocean. The channel ('Southern Ocean') extends from $68^{\circ} \mathrm{S}$ to $52^{\circ} \mathrm{S}$. The ocean has a uniform depth $D=4500 \mathrm{~m}$ except for two sills in the channel. At the sill with the Pacific (Atlantic) to its west the depth is approximately $2400 \mathrm{~m}(3400 \mathrm{~m})$. The sills are introduced to slow down the simulated Antarctic Circumpolar Current, which would otherwise be unrealistically vigorous. In addition, the sill with the Pacific to its west is required to model the Drake Passage effect (Toggweiler and Samuels, 1998). We shortly address the effect of the sills on the scaling behaviour in Section 4.1.

For our calculations we use two distinct numerical codes. Details will be given in Section 2.2, where we describe the different cases that will be explored. Both programs, however, represent the same ocean model, that is, the hydrostatic primitive equations subject to a rigid lid condition at the sea surface. The equations are expressed in pseudo-spherical $(\phi, \theta, z)$ coordinates and discretized onto an Arakawa staggered B grid. The resolution is coarse, $3.75^{\circ}$ zonally by $4^{\circ}$ meridionally. In the vertical, there are 15 layers that increase in thickness from $53 \mathrm{~m}$ at the surface to $547 \mathrm{~m}$ near the bottom. (a)

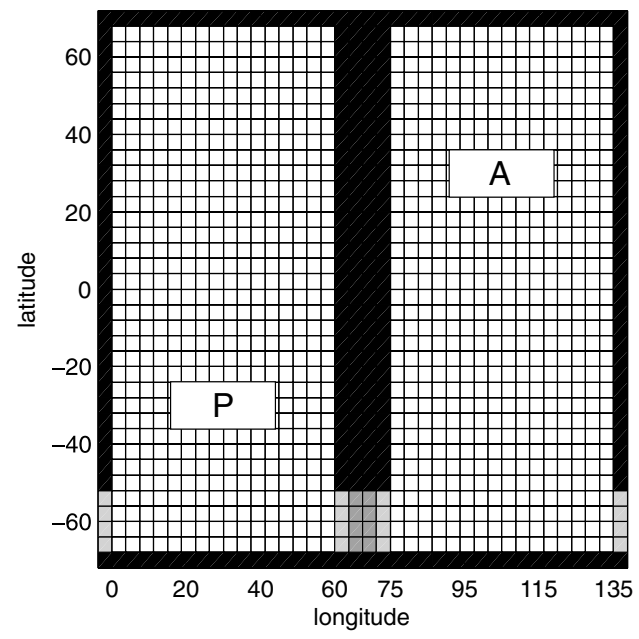

(b)

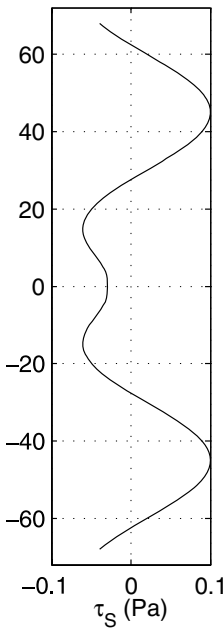

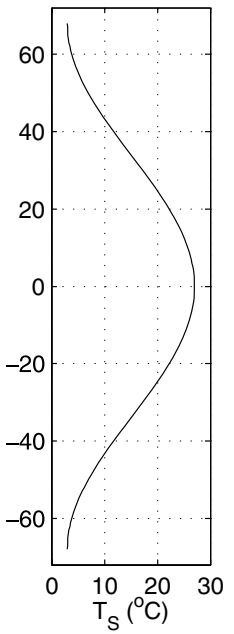

(d)

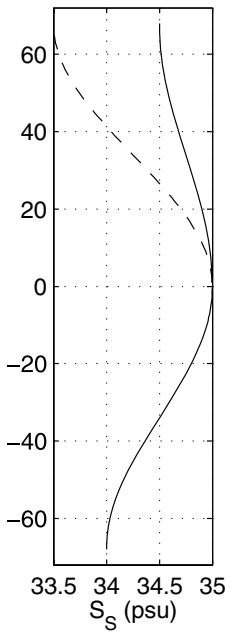

Fig. 1. (a) Model geometry. Intersections of grid lines define velocity points on the Arakawa B grid. The shading indicates depth, where white represents $4500 \mathrm{~m}$, light grey $3400 \mathrm{~m}$ and dark grey $2400 \mathrm{~m}$. The boxes labelled P and A indicate the regions in the Pacific and Atlantic across which the area-mean pycnocline depth is calculated. (b) Zonal wind stress. (c) Reference surface temperature. (d) Reference surface salinity in the Pacific (dashed line) and Atlantic (drawn line). 
Turbulent transfer of momentum is represented by the usual second-order operator with horizontal viscosity $A_{H}=2.8 \times$ $10^{5} \mathrm{~m}^{2} \mathrm{~s}^{-1}$ and vertical viscosity $A_{\mathrm{V}}=1.0 \times 10^{-3} \mathrm{~m}^{2} \mathrm{~s}^{-1}$. Free slip conditions are prescribed at the bottom boundary, while noslip conditions apply on the lateral walls. In the experiments where a wind stress is imposed, its zonal component is given by the idealized profile introduced by Bryan (1987, Fig. 1b)

$$
\begin{aligned}
\frac{\tau_{S}(\theta)}{\tau_{0}}= & 0.5(\tanh (5 \pi-10|\theta|)+\tanh (10|\theta|)) \\
& -0.8(\sin (6|\theta|)+1),
\end{aligned}
$$

where $\tau_{0}=0.1 \mathrm{~Pa}$ is a reference amplitude. The meridional wind stress is zero in all experiments.

There is, effectively, a single active tracer (density, $\rho$ ), which is subject to restoring boundary conditions at the sea surface. The relaxation time scale is $30 \mathrm{~d}$, which ensures that deviations of the surface density from the target field remain small. The target surface density is different between the Pacific and Atlantic so as to create a typical overturning pattern with interbasin exchange. This difference is more easily appreciated by specifying the target field in terms of temperature $T$ and salinity $S$, and relating those to density through a linear equation of state,

$\rho=\rho_{0}\left[1-\alpha_{T}\left(T-T_{0}\right)+\alpha_{S}\left(S-S_{0}\right)\right]$.

Here, $\rho_{0}=1035 \mathrm{~kg} \mathrm{~m}^{-3}, T_{0}=15^{\circ} \mathrm{C}$ and $S_{0}=15 \mathrm{psu}$ are reference quantities and $\alpha_{T}=1.0 \times 10^{-4} \mathrm{~K}^{-1}$ is the thermal expansion coefficient and $\alpha_{S}=7.6 \times 10^{-4} \mathrm{psu}^{-1}$ the haline contraction coefficient. The target surface temperature (Fig. 1c) is a function of latitude only,

$T_{S}(\theta)=15+12 \cos \left(\pi \frac{\theta}{\theta_{N}}\right)$,

where $\theta_{N}=68^{\circ} \mathrm{N}$. To mimic real-world conditions, the reference surface salinity (Fig. 1d) is different between the Pacific and Atlantic. It is given by

$S_{S}(\theta)=35+\chi\left[\cos \left(\pi \frac{\theta}{\theta_{N}}\right)-1\right]$,

where $\chi=0.50$ for the Southern Hemisphere, $\chi=0.75$ for the North Pacific and $\chi=0.25$ for the North Atlantic. This way, the forcing is sufficiently asymmetric to ensure that Northern Hemispheric deep water formation is confined to the Atlantic in all model runs. Note that the scaling behaviour of the Pacific and Atlantic would be nearly identical if $\chi$ would be uniform.

We explore two different parametrizations of tracer mixing by subgrid-scale motions. The first scheme uses the traditional anisotropic Fickian diffusion along horizontal/vertical directions. In the second scheme the diffusion tensor is rotated to align the dominant diffusive fluxes along the local isopycnal directions (Redi, 1982), which eliminates the strong lateral diffusion of density. Instead, the effect of mesoscale eddies on the density field is represented by the Gent \& McWilliams (1990,
GM) scheme. For the horizontal, isoneutral and GM diffusivity we use $K=1.0 \times 10^{3} \mathrm{~m}^{2} \mathrm{~s}^{-1}$ throughout, but the latter two are tapered to zero in regions of steep isoneutral slopes using the scaling proposed by Gerdes et al. (1991). Tracer advection is discretized with a second order centred difference scheme. Convection is parametrized by employing a strongly enhanced vertical diffusivity $\left(K_{\mathrm{V}}^{c}=1.0 \mathrm{~m}^{2} \mathrm{~s}^{-1}\right)$ at interfaces across which the density gradient is statically unstable. Lateral walls and bottom are assumed insulating.

\subsection{Experiments}

We perform four scaling experiments that differ from each other by the use of HORizontal or GM tracer mixing, and by the absence (-NW) or presence of wind stress: HOR-NW, HOR, GM-NW and GM. In each case we need to solve the steady state problem

$\mathbf{F}\left(\overline{\mathbf{u}}, K_{\mathrm{V}}\right)=\mathbf{0}$,

for a range of values of $K_{\mathrm{V}}$. Here, $\mathbf{F}$ denotes the discrete operator of the right hand side of the evolution equations of the ocean model, and $\overline{\mathbf{u}}$ is the model state vector.

The HOR-NW and HOR experiments use the B-grid version of the ThermoHaline Circulation Model (De Niet et al., 2007, THCM) to solve eq. (6). THCM is a fully implicit model, capable to compute branches of exact steady states using continuation methods (Dijkstra, 2005). Given a suitable prediction for a steady state $\overline{\mathbf{u}}$, for example the previous solution on the branch, the model evaluates both $\mathbf{F}$ and the Jacobian matrix of $\mathbf{F}$ and subsequently uses the Newton-Raphson method to converge to $\overline{\mathbf{u}}$. The linear systems are solved with an iterative solver using a (tailored) preconditioning technique. The relatively low computational cost of implicit modelling allows to explore a very wide range of vertical diffusivities in an efficient manner. However, THCM only operates effectively when using a horizontal tracer mixing scheme.

For the GM-NW and GM experiments, in which we reevaluate the scaling behaviour using the isopycnal/GM tracer mixing scheme, we therefore resort to the familiar GFDL Modular Ocean Model (MOM), version 3.1 (Pacanowski and Griffies, 2000). With MOM, (approximate) steady states are obtained by integrating forward in time until some norm $\|\mathbf{F}\|$ is acceptably small; we stop the integration as soon as the basin averaged surface heat flux drops below $10^{-3} \mathrm{~W} \mathrm{~m}^{-2}$. The typical equilibration time is determined by the ratio of the depth scale squared to diapycnal diffusivity, which amounts to $\mathcal{O}\left(10^{4}\right)$ years. Obviously, the large computational expense limits the number of equilibrium solutions that can be found. In the GM-NW and GM experiments nine points are computed with diffusivities varying over two orders of magnitude, ranging from $K_{\mathrm{V}}=0.053$ to $5.3 \mathrm{~cm} \mathrm{~s}^{-1}$. 


\section{Scaling behaviour}

In this section, we focus on measures of the overturning rate $\Psi$ and pycnocline depth $\delta_{\rho}$ in order to assess the scaling behaviour of the circulation in the simple global geometry. The overturning rate is monitored separately for the Atlantic and Pacific by determination of the maximum (absolute value of the minimum) of the stream function in the Atlantic (Pacific). The stream function is calculated with $z$ as a vertical coordinate. In the cases where a wind stress is imposed only the extrema occurring below a depth of $500 \mathrm{~m}$ are considered so that contributions from the shallow wind-driven cells are excluded.

Similar to Dijkstra (2008), the measure of the pycnocline depth is determined by calculating the depth where the difference between in situ and surface density is equal to a fraction $\eta$ of the difference between bottom and surface density:

$\rho\left[z=\delta_{\rho} \log (1-\eta)\right]-\rho_{S}=\eta\left(\rho_{\mathrm{B}}-\rho_{\mathrm{S}}\right)$,

where $\rho_{\mathrm{S}}\left(\rho_{\mathrm{B}}\right)$ is the density of the surface (bottom) grid cell. The sensitivity of $\delta_{\rho}$ to $K_{\mathrm{V}}$ was shown to be independent of the value of $\eta$ (Dijkstra, 2008) and here we choose $\eta=0.75$. Note that the resulting depth is rescaled by $\log (1-\eta)$, so that $\delta_{\rho}$ is equal to the e-folding length scale in case the density profile is exponential and $\delta_{\rho} / D \ll 1$. Obviously, the choice of the measure of the pycnocline depth is not unique. For example, Park and Bryan (2000) used an integral measure. However, several tests have indicated that the use of their depth scale instead of $\delta_{\rho}$ as defined in eq. (7) does not alter our results significantly. In the following, we present area-averaged pycnocline depths for two regions (each $30^{\circ}$ in longitude $\times 12^{\circ}$ in latitude), one centred in the South Pacific and the other in the North Atlantic subtropical gyre (Fig. 1a).

Anticipating power law scaling behaviour, the response of the solution to changing vertical diffusivity is quantified by the

(a)

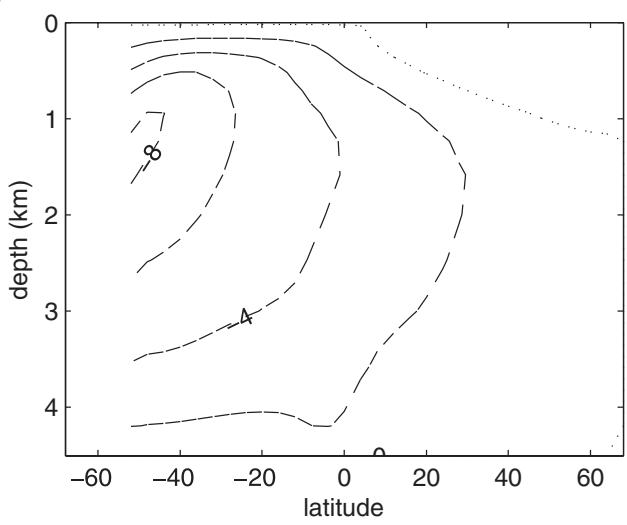

logarithmic sensitivity, which for a metric $X$ is defined as

$\frac{\partial \log X}{\partial \log K_{\mathrm{V}}}=\frac{K_{\mathrm{V}}}{X} \frac{\partial X}{\partial K_{\mathrm{V}}}$.

In the following, 'sensitivity' should be interpreted with reference to eq. (8).

\subsection{HOR-NW experiment}

Without wind forcing $\left(\tau_{0}=0.0 \mathrm{~Pa}\right)$, the structure of the overturning circulation is controlled only by the upper boundary condition for density [eqs (4) and (5)]. As shown in Fig. 2 for $K_{\mathrm{V}}=0.53 \mathrm{~cm} \mathrm{~s}^{-1}$, it crudely resembles the global "conveyor' circulation seen in more advanced models and is typical for this geometry (Von der Heydt and Dijkstra, 2007). The flow in the Atlantic is characterized by a net downward motion between $50^{\circ} \mathrm{N}$ and $68^{\circ} \mathrm{N}$, which is compensated for by the sum of net upwelling within the basin and transport across the southern boundary. In the Pacific the zonally integrated motion is upward throughout the basin for depths below $1 \mathrm{~km}$.

Using THCM a branch of steady states is computed for diffusivities ranging from $K_{\mathrm{V}}=0.0053$ to $5.3 \mathrm{~cm} \mathrm{~s}^{-1}$, that is, $K_{\mathrm{V}}$ is varied over three orders of magnitude. In both basins the scaling behaviour is close to the classical predictions for the lower range of diffusivities: the sensitivity of the overturning rates is $2 / 3$, while the pycnocline depths show a $1 / 3$ power law dependence (Fig. 3). For higher diffusivities the results deviate from these relations. The Atlantic overturning strength is clearly less sensitive for diffusivities larger than about $0.1 \mathrm{~cm}^{2} \mathrm{~s}^{-1}$ and follows an approximate 1/3-dependence for the upper range of diffusivities. Although only slightly, the scaling of the Pacific overturning rate and the pycnocline depths also depart from the classical behaviour for $K_{\mathrm{V}} \gtrsim$ $1.0 \mathrm{~cm}^{2} \mathrm{~s}^{-1}$.

(b)

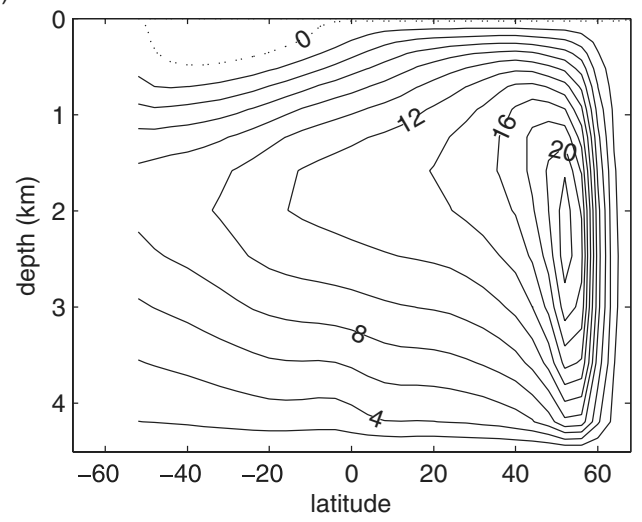

Fig. 2. Contour plots of the (a) Pacific and (b) Atlantic meridional overturning stream functions for $K_{\mathrm{V}}=0.53 \mathrm{~cm} \mathrm{~s}^{-1}$ in the HOR-NW experiment. The contour interval is $2 \mathrm{~Sv}$. 
(a)

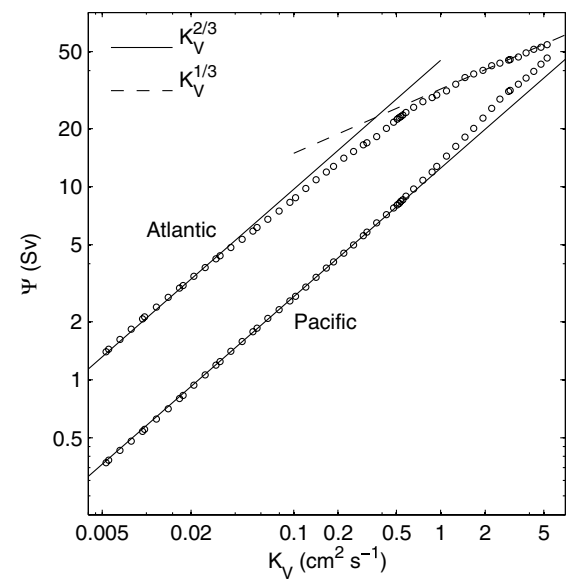

(b)

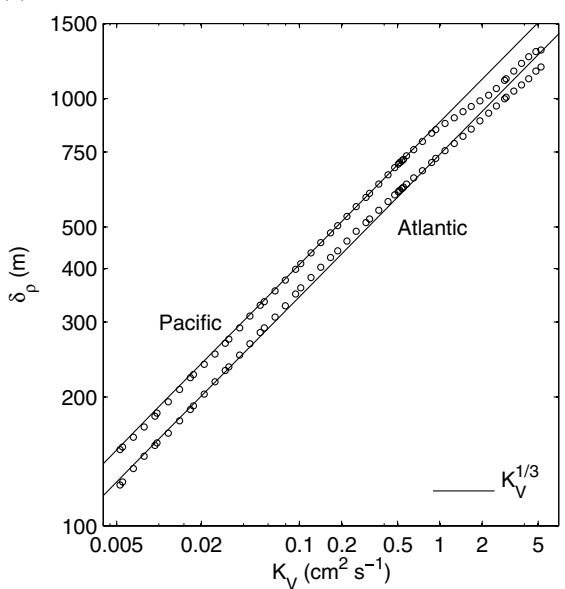

Fig. 3. Scaling behaviour in the HOR-NW experiment. Each symbol corresponds to an exact steady state obtained with THCM. The lines are drawn for reference only and do not correspond to regressions. (a) Overturning strength. (b) Pycnocline depth.

\subsection{HOR experiment}

Starting from a HOR-NW solution we can easily find a corresponding solution with full wind forcing by continuation in $\tau_{0}$ from 0.0 to $0.1 \mathrm{~Pa}$. At $K_{\mathrm{V}}=0.53 \mathrm{~cm} \mathrm{~s}^{-1}$ the result is a linear increase in the pycnocline depths, which is in contrast with the scaling relations for a single-hemispheric basin put forward by Welander (1971) and Vallis (2000) that predict a square root dependence. The Atlantic overturning strength also increases with wind strength, but without an evident relation between the two, while the Pacific overturning rate remains almost constant. The resulting overturning pattern mainly differs from that in the HOR-NW case by the presence of shallow (less than $500 \mathrm{~m}$ deep) Ekman cells.

Using the full wind forcing [eq. (2)], we again compute a branch of steady solutions with $K_{\mathrm{V}}$ as control parameter.
Figure 4 shows that the scaling behaviour in the HOR experiment differs significantly from that in the HOR-NW experiment. The solution's sensitivity to $K_{\mathrm{V}}$ is very small in the lower diffusivity range. With increasing diffusivity the sensitivity of the overturning strength increases to about $1 / 3$ for the Atlantic and to somewhat more than $2 / 3$ for the Pacific. At higher diffusivities the sensitivity of the pycnocline depth is slightly larger than 1/6 for both the Atlantic and the Pacific. So, similar to the results of Dijkstra (2008), the scaling exponents for $\Psi_{\mathrm{A}}$ and $\Psi_{\mathrm{P}}$ are different across a large part of the range of diffusivities. The sensitivity to $K_{\mathrm{V}}$ of the pycnocline depth, however, is the same in the Pacific and the Atlantic.

Anticipating the use of MOM for the GM-NW and GM experiments, we point out that results from THCM are consistent with results from MOM. Superimposed on the exact steady states from THCM, Fig. 4 shows four larger symbols corresponding (a)

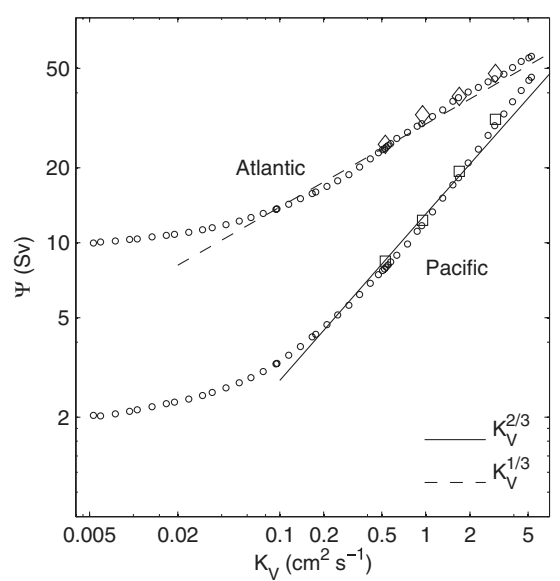

(b)

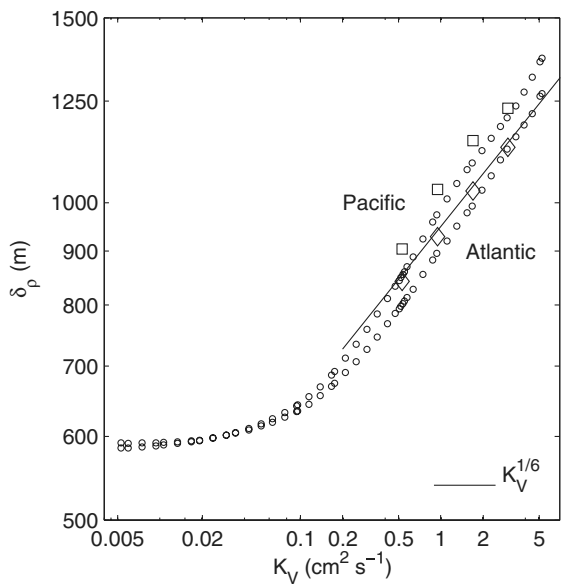

Fig. 4. Scaling of the (a) overturning strength and (b) pycnocline depth in the HOR experiment. The small symbols represent THCM solutions, while the four larger symbols correspond to solutions from MOM. 
to MOM solutions for the HOR case (for $K_{\mathrm{V}}=0.53,0.95,1.7$ and $3.0 \mathrm{~cm}^{2} \mathrm{~s}^{-1}$ ). In addition to the good agreement between the results, we note that the $K_{\mathrm{V}}$-spacing (an quarter of an order of magnitude) between the successive MOM solutions is sufficient to detect changes in the sensitivity.

\subsection{GM-NW experiment}

The typical pattern of the overturning circulation in the GM-NW experiment is plotted in Figs 5a and b, which shows the circulation for $K_{\mathrm{V}}=0.53 \mathrm{~cm} \mathrm{~s}^{-1}$. Compared to the HOR-NW case there is a strong reduction in Eulerian-mean overturning in the North Atlantic, while the export across the southern boundary is almost unaltered (Fig. 5c). The change in overturning is rather small in the Pacific and therefore not shown. In addition to the Eulerian-mean overturning there is a significant eddy-induced component (Fig. 5d), although it is largely confined to the surface. The eddy-induced circulation is most pronounced in the Southern Ocean and in the North Atlantic.

The scaling plots for this experiment are presented in Fig. 6. The left-hand panel shows the overturning rate based on Eulerian-mean transports as well as with the eddy-induced component included. The Eulerian-mean and residual MOC strength are nearly identical for the Pacific. For the Atlantic the difference between the two is small, because there is no significant eddy-induced transport at the depth of maximum Eulerian-mean transport (compare Figs $5 \mathrm{~b}$ and d), and the sensitivity to changes in $K_{\mathrm{V}}$ is the same. The scaling of both overturning strength and pycnocline depth is only weakly affected by the change from the horizontal to the isopycnal/GM representation of lateral mixing. As in the HOR-NW experiment the results depart from the classical scaling relations only for the higher range of diffusivities. Yet, the diffusivity up to which classical scaling holds, appears to be higher for GM-NW than for HOR-NW.

\subsection{GM experiment}

Similar to the result in the case of horizontal mixing, comparison of GM-NW and GM solutions indicates that the presence of wind forcing leads to an increase in pycnocline depth. Except for the emergence of shallow Ekman cells, the pattern of the overturning circulation is not significantly altered. At $K_{\mathrm{V}}=0.53 \mathrm{~cm} \mathrm{~s}^{-1}$ the difference between the HOR and GM solution is comparable to the difference between the HOR-NW and GM-NW solution (Fig. 5c). Furthermore, as in the GM-NW case (Fig. 5d), the eddy-induced circulation is most intense close to surface and mainly present in the Southern Ocean and North Atlantic.

Figure 7 shows the scaling behaviour for this experiment. The sensitivity to $K_{\mathrm{V}}$ of the Pacific overturning strength appears to be constant across the range of diffusivities and is somewhat larger (a)

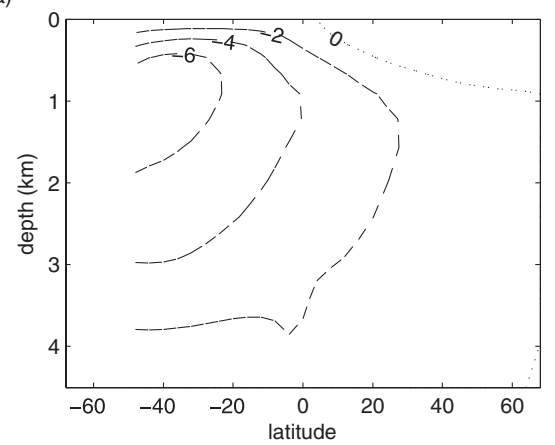

(c)

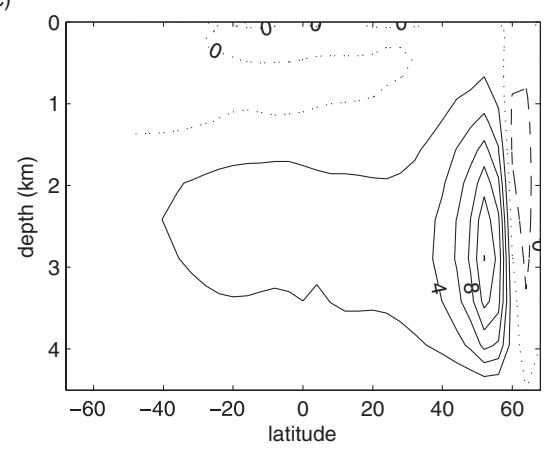

(b)

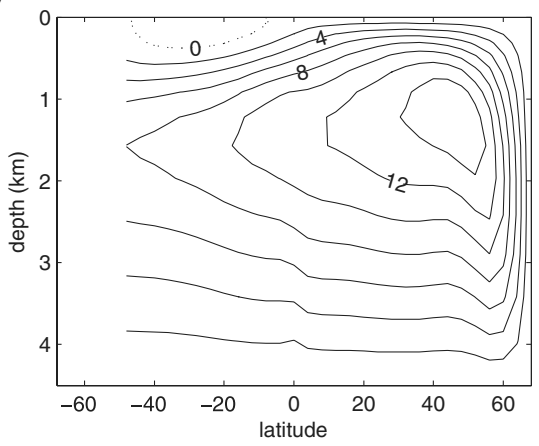

(d)

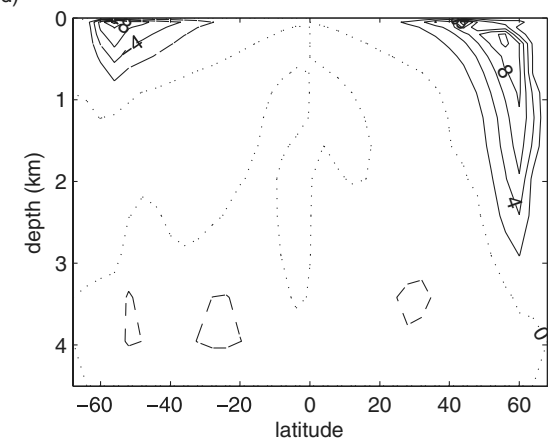

Fig. 5. (a,b) Contour plots of the (a) Pacific and (b) Atlantic (Eulerian-mean) meridional overturning in the GM-NW experiment. (c) Difference in Atlantic meridional overturning circulation between HOR-NW and GM-NW. (d) Eddy-induced global overturning in the GM-NW experiment. All plots are for $K_{\mathrm{V}}=0.53 \mathrm{~cm} \mathrm{~s}^{-1}$ and have a contour interval of $2 \mathrm{~Sv}$. 
(a)

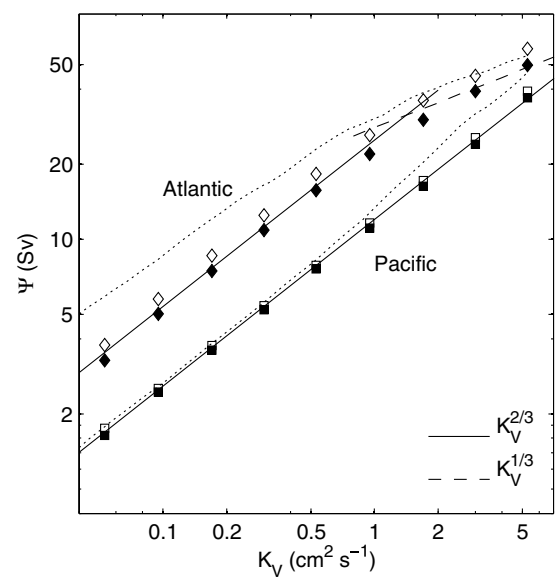

(b)

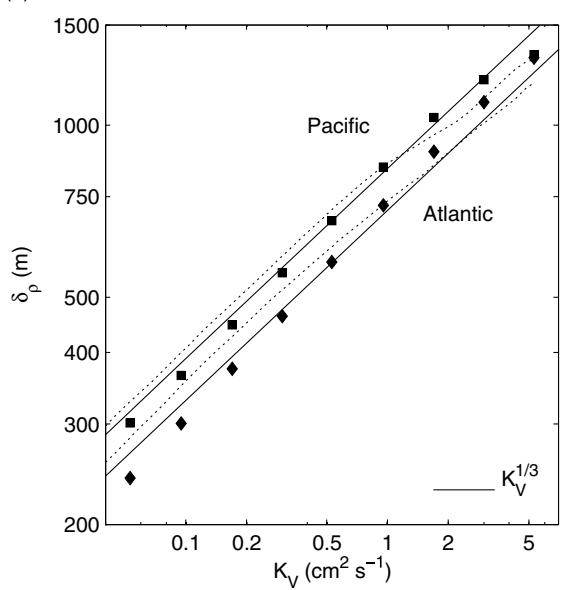

Fig. 6. Scaling behaviour in the GM-NW experiment. The symbols represent (quasi-)steady states obtained with MOM. For comparison, the dotted lines show the results from the HOR-NW experiment. (a) Eulerian-mean (solid symbols) and residual (open symbols) overturning strength. (b) Pycnocline depth.

(a)

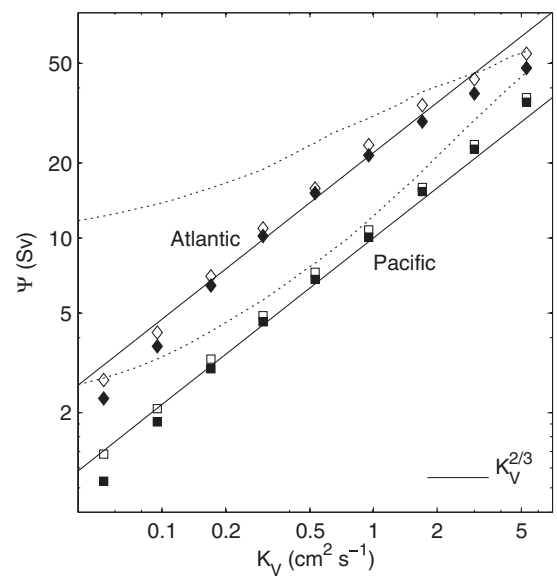

(b)

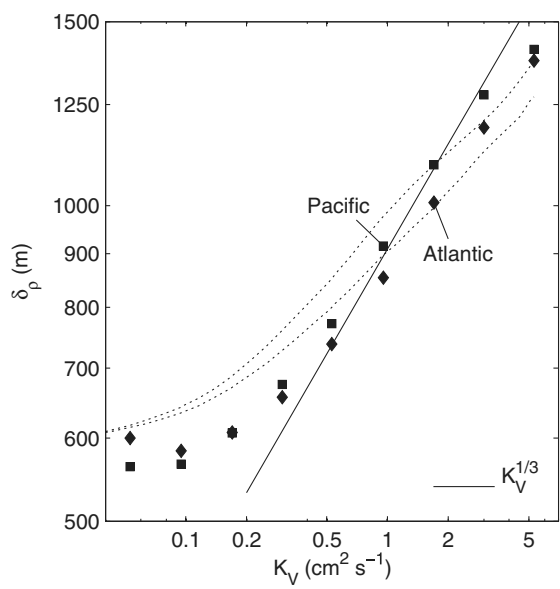

Fig. 7. Scaling behaviour as in Fig. 6, but now for the GM experiment. Here, the dotted lines show the results from the HOR experiment.

than $2 / 3$. The sensitivity of the Atlantic overturning is similar for low diffusivities, but decreases as $K_{\mathrm{V}}$ increases. The pycnocline depth is again of comparable magnitude in the two basins. The sensitivity of $\delta_{\rho}$ to changes in $K_{\mathrm{V}}$ increases with increasing $K_{\mathrm{V}}$, but remains clearly smaller than $1 / 3$. Comparison of Figs 6 and 7 demonstrates that the scaling of the overturning strength is much less affected by the change from horizontal to isopycnal/GM mixing in absence of wind stress than in presence of wind stress. Furthermore, the scaling of the pycnocline depth is strongly influenced by the presence of wind for both representations of lateral mixing.

\section{Analysis}

In the following we analyse the results of Section 3 more closely with the help of two questions: (1) What explains the departure from the classical scaling relations in the experiments without wind? and (2) Why does the representation of lateral mixing have so much impact on the scaling when wind is present?

\subsection{Deviations from classical scaling in the absence of wind}

The results from both the HOR-NW and GM-NW experiments indicate that, at least for larger diffusivities, the relation between overturning strength and pycnocline depth is not the same for the Pacific and Atlantic system. Two possible relations between $\Psi$ and $\delta_{\rho}$ are suggested by classical scaling theory, the first expressing the thermal wind balance $\left(\Psi \propto \delta_{\rho}^{2}\right)$ and the second the vertical advection-diffusion balance $\left(\Psi \propto K_{\mathrm{V}} / \delta_{\rho}\right)$. The classical scaling laws hold provided that both relations are 
(a)

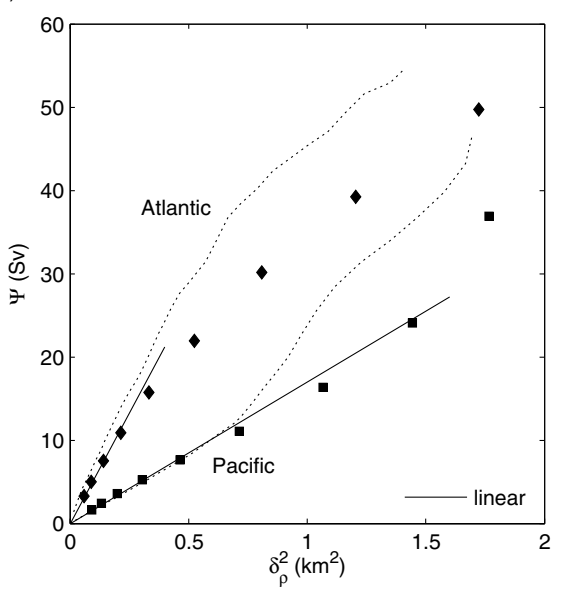

(b)

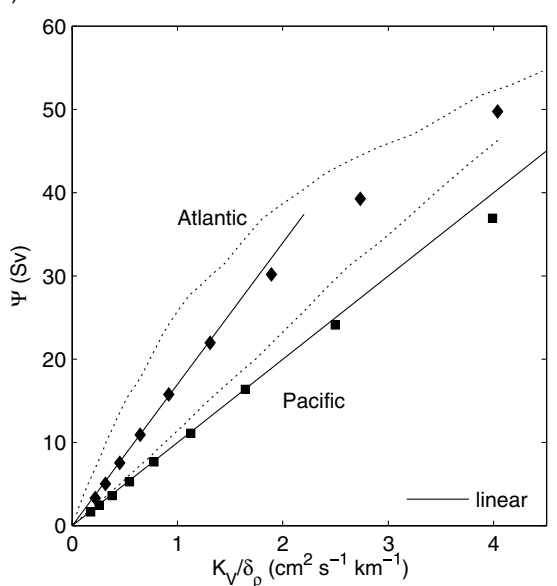

Fig. 8. Comparison of the results of the 'no wind' experiments with predictions from the (a) 'thermal wind' and (b) 'advection-diffusion balance' scaling relation. Dotted lines represent HOR-NW and symbols GM-NW.

satisfied simultaneously [eq. (1)], which is indeed the case for small overturning rates (small $K_{\mathrm{V}}$, Fig. 8). For higher diffusivities both relations predict stronger Atlantic overturning than observed in the model. The Pacific overturning is consistent with the advection-diffusion balance relation across the entire range of $K_{\mathrm{V}}$, but at higher diffusivities stronger than predicted by the thermal wind relation. It is remarkable that the HOR-NW solutions for which classical scaling holds, overlap with the GMNW solutions in the $\Psi$ versus $\delta_{\rho}^{2}$-plane, but indicate a stronger derivative in the $\Psi$ versus $K_{\mathrm{V}} \delta_{\rho}^{-1}$-plane. This suggests that, in absence of wind, the effect of spurious diapycnal mixing due to horizontal diffusion is in some sense equivalent to enhancing vertical diffusion by multiplication of $K_{\mathrm{V}}$ with a certain factor.

While it is true that (locally) the stratification is also determined by processes other than vertical advection and diffusion, the results above do not mean that the large-scale flow is no longer in geostrophic and hydrostatic balance as the scaling starts to depart from the classical relations. Instead, the 'thermal wind relation' between $\Psi$ and $\delta_{\rho}$ does not adequately capture that the flow is in thermal wind balance. So far, however, the analysis failed to take into account that the density contrast $\Delta \rho$ in eq. (1) may depend on $K_{\mathrm{V}}$. Even if using strong restoring conditions, slight variations in the surface density field occur due to changes in surface advection. Park and Bryan (2000) showed that the scaling behaviour of single-hemispheric flows is closer to the classical relations when the results are corrected for this effect.

We first apply this idea inversely, by calculating the variation in $\Delta \rho$ required to compensate for the departure from classical scaling. The strongest variations in the required (compensating) density contrast are found when it is calculated from the overturning strength as

$\Delta \rho \propto \Psi^{3} K_{\mathrm{V}}^{-2}$.
As expected, the Atlantic overturning rate in the HOR-NW experiment requires the largest variation in $\Delta \rho$ (Fig. 9a): between $K_{\mathrm{V}}=0.053$ and $5.3 \mathrm{~cm}^{2} \mathrm{~s}^{-1}$ the density contrast should decrease by one order of magnitude to account for the departure of the scaling of $\Psi_{\mathrm{A}}$ from the classical relations. At high diffusivities the required sensitivity of $\Delta \rho$ in the Atlantic is about -1 for both HOR-NW and GM-NW. Such variations are much larger than can be realized when using strong restoring conditions. Compensation of the scaling of the Pacific overturning is only needed in the HOR-NW experiment. In that case a small positive sensitivity of $\Delta \rho$ would be required.

It is not straightforward to determine the actual variation in density contrast. When using the difference between sectionaveraged densities at two latitudes, as in Dijkstra (2008), the result is very sensitive to the choice of latitudes and the depth range over which the average is calculated. We found that a more robust measure of $\Delta \rho$ can be determined from the basin-wide meridional buoyancy transport

$B^{\theta_{\mathrm{S}}}=\iint_{\theta_{\mathrm{S}}} v \rho \mathrm{d} \phi \mathrm{d} z$

where the integral is taken across a zonal section at latitude $\theta_{S}$. We then define the actual density difference as the ratio of equatorial buoyancy transport and overturning strength,

$\Delta \rho=-\frac{B^{0}}{\Psi}$.

The result can be thought of as the effective density contrast carried by the overturning and is plotted in Fig. 9b. The strongest variations in the actual density contrast are found in the HOR-NW experiment. In the Pacific $\Delta \rho$ decreases by a factor two between $K_{\mathrm{V}}=0.053$ and $5.3 \mathrm{~cm}^{2} \mathrm{~s}^{-1}$; at high diffusivities the sensitivity is about $-1 / 6$. In the GM-NW experiment the sensitivities are even smaller (less negative). These results imply that only a fraction of the departure from classical scaling 
(a)

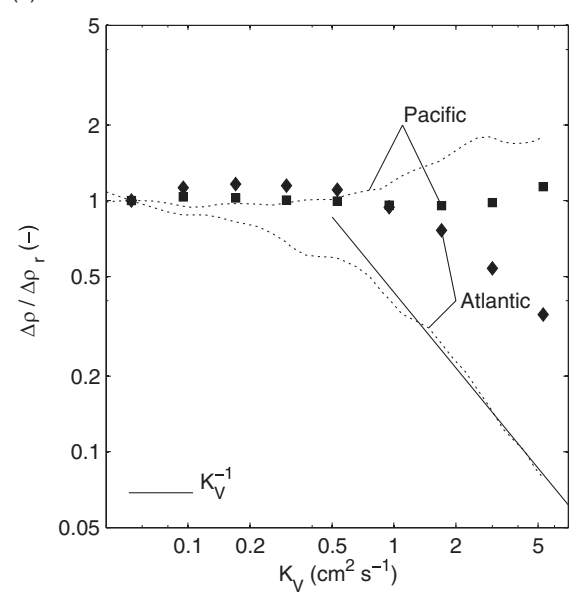

(b)

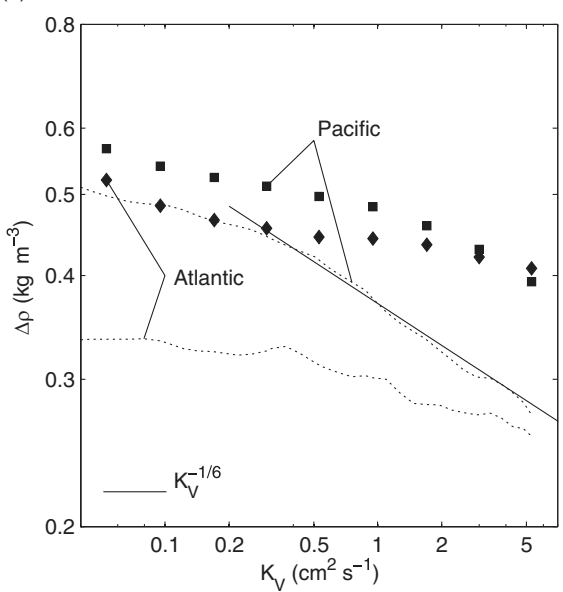

Fig. 9. Variations in the density contrast $\Delta \rho$ in the HOR-NW (dotted lines) and GM-NW (symbols) experiments. (a) Density contrast required to account for the difference between the actual scaling of the overturning rate and the classical 2/3-dependence. The results are normalized by a reference contrast $\Delta \rho_{r}=\Delta \rho\left(K_{\mathrm{V}}=0.053 \mathrm{~cm}^{2} \mathrm{~s}^{-1}\right)$. (b) Density contrast, as determined by division of the basin-wide equatorial buoyancy transport $B^{0}$ by the overturning strength $\Psi$.

of the Atlantic overturning strength can be compensated for by accounting for variations in the density contrast. For the Pacific compensation by $\Delta \rho$ would even cause a stronger departure from classical scaling.

The break down of the scaling thus has a more fundamental cause. One of the assumptions in classical scaling theory is that the ocean is very deep compared to the characteristic depth scale of the stratification. Obviously, the validity of this assumption decreases as $K_{\mathrm{V}}$ increases. We therefore examine changes in the vertical structure of the flow. Figure 10a shows the meridional overturning in the Atlantic at $K_{\mathrm{V}}=5.3 \mathrm{~cm} \mathrm{~s}^{-1}$ in the GM-NW experiment. It is clear that the depth scale of the flow is increased compared to the solution at $K_{\mathrm{V}}=0.53 \mathrm{~cm} \mathrm{~s}^{-1}$ (Fig. 5b). To allow a more quantitative analysis we define the flow-based depth scale $\delta_{v}$ as the depth where the zonally integrated velocity is zero, that is, the depth of maximal (in absolute sense) overturning. We calculate $\delta_{v}$ at $30^{\circ} \mathrm{S}$ in the Pacific and at $30^{\circ} \mathrm{N}$ in the Atlantic and compare the result to the pycnocline depth scale $\delta_{\rho}$ (Fig. 10b). In the Pacific the agreement between the two depth scales is very good, especially in the GM-NW experiment. In the Atlantic, however, there is only a linear relation between the two scales for low diffusivities, in which case the flow based depth scale is much larger than the pycnocline depth. With increasing diffusivity the increase in $\delta_{\rho}$ is stronger than the increase in $\delta_{v}$.

The divergence of the two depth scales is associated with changes in the depth-zonal structure of the meridional velocity (Figs 10c-e). For all diffusivities, the northward surface transport is carried by a strong western boundary current, while the return transport at depth is associated with weaker velocities. For small values of $K_{\mathrm{V}}$ the zonal structure of the meridional velocities is relatively simple; across the largest part of the depth range they change sign only once between west and east. With increasing diffusivities the velocity pattern deepens and the zonal structure becomes more complicated. The surface western boundary current connects with a deep patch of northward velocities, which is present to the east of the deep western boundary current. The southward velocities to the east of the surface boundary current are also part of a patch that extends to the bottom. These results suggest that the divergence of $\delta_{\rho}$ and $\delta_{v}$ in the Atlantic is symptomatic for changes in the zonal structure of the solution. Classical scaling theory, however, does not account for such changes.

When comparing the Atlantic overturning in the GM-NW experiment between $K_{\mathrm{V}}=0.53 \mathrm{~cm} \mathrm{~s}^{-1}$ (Fig. 5b) and $K_{\mathrm{V}}=$ $5.3 \mathrm{~cm} \mathrm{~s}^{-1}$ (Fig. 10a), the increase in depth scale appears to be stronger in the southern part than in the northern part of the basin. As a result, the fraction (in a zonally integrated sense) of the Atlantic deep water that is transported across the southern boundary changes as the scaling starts to deviate from the classical relations. For diffusivities up to $K_{\mathrm{V}}=0.53 \mathrm{~cm} \mathrm{~s}^{-1}$ about 52 per cent of the deep water is exported. This fraction decreases towards higher diffusivities and equals about 38 per cent for $K_{\mathrm{V}}=$ $5.3 \mathrm{~cm} \mathrm{~s}^{-1}$. Similar changes occur in the HOR-NW experiment, in which case the exported fraction of Atlantic deep water varies between 30 and 40 per cent up to $K_{\mathrm{V}} \approx 2 \mathrm{~cm}^{2} \mathrm{~s}^{-1}$, but decreases sharply to below 20 per cent at $K_{\mathrm{V}}=5.3 \mathrm{~cm} \mathrm{~s}^{-1}$. In this case, there are also changes in the structure of the Pacific overturning. For diffusivities larger than about $1 \mathrm{~cm}^{2} \mathrm{~s}^{-1}$ a part of the upwelled water sinks at the southern edge of the Pacific (about 20 per cent in a zonally integrated sense at $K_{\mathrm{V}}=5.3 \mathrm{~cm} \mathrm{~s}^{-1}$ ). So, in general, the interbasin exchange tends to decrease with increasing $K_{\mathrm{V}}$, because the typical 'conveyor' circulation cannot be maintained in the diffusive limit. 
(a)

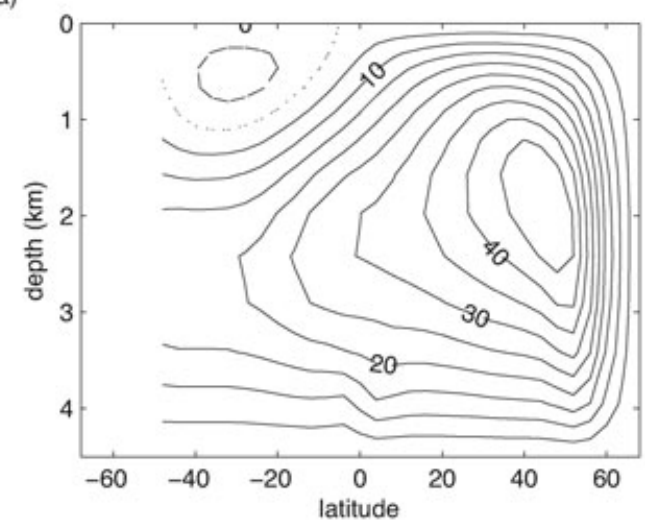

(c)

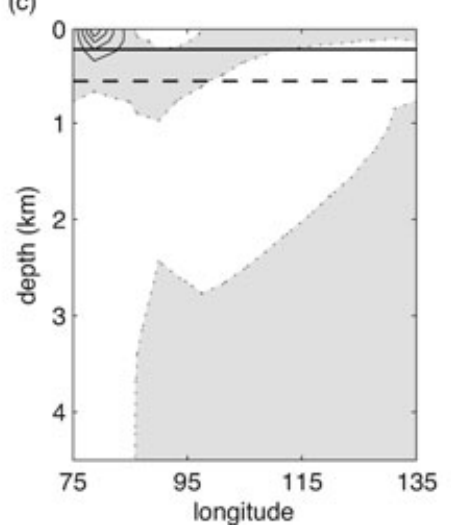

(d)

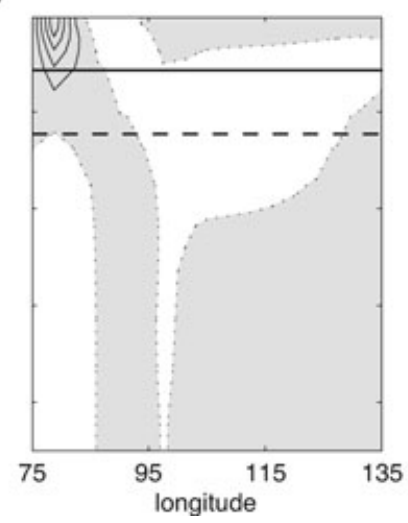

(b)

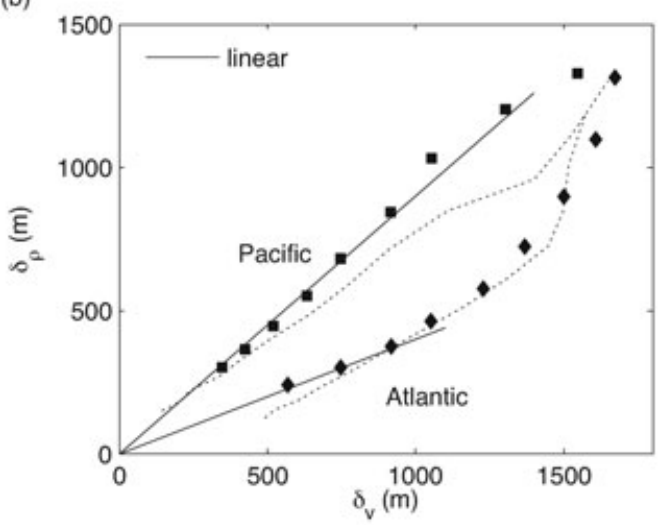

(e)

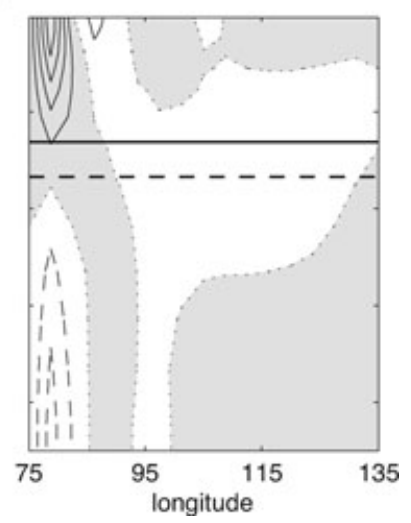

Fig. 10. Analysis of changes in the structure of the meridional velocity. (a) Contour plot (interval $5 \mathrm{~Sv}$ ) of the Atlantic meridional overturning for $K_{\mathrm{V}}=5.3 \mathrm{~cm} \mathrm{~s}^{-1}$ in the GM-NW experiment. (b) Comparison of the flow-based depth scale $\delta_{v}$ to the pycnocline depth $\delta_{\rho}$ in the HOR-NW (dotted lines) and GM-NW (symbols) experiments. (c-e) Zonal cross-sections at $30^{\circ} \mathrm{N}$ of Atlantic meridional velocity for (c) $K_{\mathrm{V}}=0.053$, (d) 0.53 and (e) $5.3 \mathrm{~cm}^{2} \mathrm{~s}^{-1}$ in the GM-NW experiment. The velocities are normalized by the maximum value in each section. Shading indicates northward flow and the contour interval is 0.2 . The drawn (dashed) line represents the level of the pycnocline depth $\delta_{\rho}$ (flow based depth scale $\delta_{v}$ ).

A natural question is if the presence of sills in the Southern Ocean interferes with this exchange as the depth scale of the flow increases, thereby changing the scaling behaviour. Using THCM we therefore repeat the HOR-NW experiment, but without sills. Figure 11 shows that the break down of the scaling of the Atlantic overturning strength is hardly affected if the sills are removed. Yet, different from the case with sills (Fig. 3a), the Pacific and Atlantic overturning strength converge rapidly for diffusivities larger than about $1 \mathrm{~cm}^{2} \mathrm{~s}^{-1}$. As a result, the sensitivity of $\Psi_{\mathrm{P}}$ to changes in $K_{\mathrm{V}}$ is about $1 / 3$ for the highest diffusivities considered, implying that the scaling of the Pacific and Atlantic overturning strengths is again almost symmetric. In this regime, however, the export (in a zonally integrated sense) of Atlantic deep water to the Southern Ocean has vanished. The pattern of the overturning circulation is fundamentally different from the typical solutions considered so far and is characterized by a roughly equatorially symmetric circulation in each basin. The scaling being similar is therefore probably not explained by enhanced interbasin exchange, but rather by the similarity of the circulation pattern. The departure of the pycnocline scaling from the classical 1/3-dependence is still present in the absence of sills. So, in the HOR-NW experiment, and probably also in GM$\mathrm{NW}$, the scaling is not affected by the presence of the sills, except when approaching a regime that is irrelevant to oceanographic modelling.

\subsection{Impact of spurious diapycnal mixing in presence of wind}

In order to evaluate if parts of the classical scaling theory still hold in presence of wind stress, Fig. 12 presents the thermal wind and advection-diffusion relations between $\Psi$ and $\delta_{\rho}$. Regarding the GM results, we note that, as in the GM-NW experiment, the advection-diffusion balance relation is well satisfied at all diffusivities for the Pacific, and also shows consistency for the Atlantic at low diffusivities. In neither basin, however, are the results consistent with the thermal wind relation. This finding disagrees with the thermocline theory of Gnanadesikan (1999), who assumed that the rate of deep water formation is proportional to the square of the thermocline depth. 


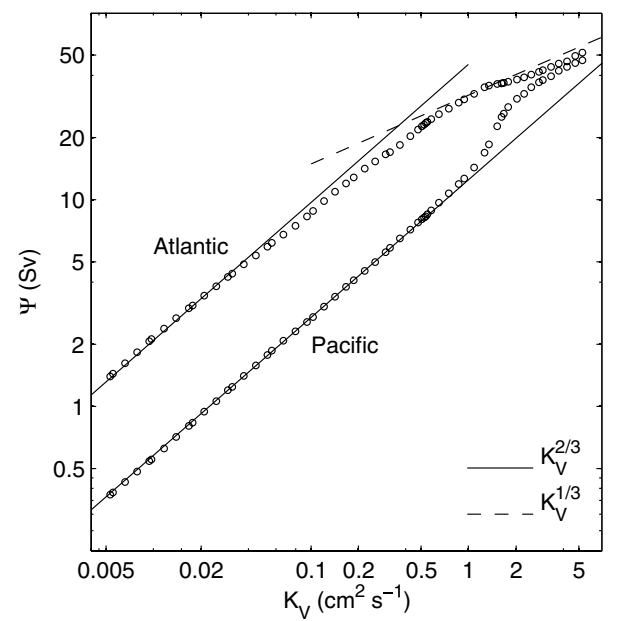

Fig. 11. Scaling of the overturning strength in an experiment with the same configuration as HOR-NW, but without sills in the Southern Ocean.

In contrast to the 'no wind' case, the relations differ markedly between the two parametrizations of lateral mixing, notably the advection-diffusion relation in the Atlantic, which does not hold for any diffusivity in the HOR experiment. In the following we examine this difference more closely by comparing the sensitivities of the overturning strength and pycnocline depth between the two experiments.

The contrast between HOR and GM is most apparent in the difference in $\Psi$ at low diffusivities (Fig. 7a). The rather large overturning rate in HOR can be understood by inspection of the circulation pattern for $K_{\mathrm{V}}=0.0053 \mathrm{~cm} \mathrm{~s}^{-1}$ (Fig. 13). At this diffusivity, only the Atlantic exhibits a deep basin-wide overturning circulation. In a zonally integrated sense about $10 \mathrm{~Sv}$ of deep water is formed at high latitudes. This is partly compensated for by upwelling outside the Atlantic and subsequent

(a)

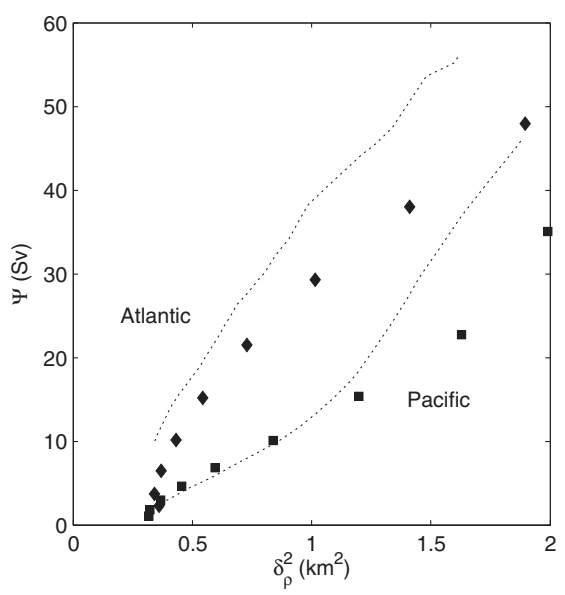

transport by the Ekman flow at the southern margin of the basin, but most of the upwelling takes place north of the equator and is concentrated in a narrow patch adjacent to the western boundary. This strongly suggests that the net upward motion is due to false diapycnal fluxes induced by horizontal diffusion across the sloping isopycnals of the boundary current ('Veronis effect'; Veronis, 1975). When using the isopycnal/GM formulation of lateral mixing, the western boundary upwelling does not vanish. The Veronis (1975) effect is absent, however, because the upwelling cancels exactly with its companion interior downwelling upon integration over the basin width and hence does not project onto the MOC (Lazar et al., 1999).

It is thus clear that the MOC scaling in the HOR experiment is strongly influenced by the incorrect representation of mixing processes. Yet, it was noted above that the spurious mixing does not preclude classical scaling behaviour if the wind forcing is zero. In that case the flow is entirely buoyancy-driven. If wind is present, on the other hand, Ekman pumping additionally drives gyre circulations that are intensified close to the western boundary. Although density and wind-driven motions interact with each other, the strength of the gyres is to a certain extent independent of vertical diffusivity. We therefore hypothesize that the wind-driven circulation imposes additional external constraints on the density field, which prevents the effect of spurious mixing from being expressed as the equivalent of enhanced vertical diffusivity.

As an aside, we note that Fig. 13 also reveals another artificial feature of the circulation, which is related to the vertical resolution of the model. As demonstrated by Weaver \& Sarachik (1990), the cells that occur to the north and south of the equator represent a numerical artefact that is present when the stability conditions on the vertical grid Reynolds and Péclet numbers are violated. Indeed, their amplitude diminishes with increasing $K_{\mathrm{V}}$ (decreasing Péclet number) up to about $0.015 \mathrm{~cm}^{2} \mathrm{~s}^{-1}$, where

(b)

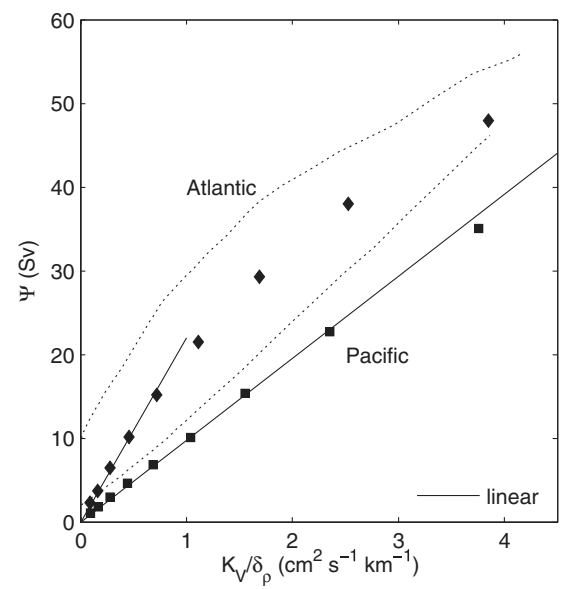

Fig. 12. Same as Fig. 8, but for the experiments with non-zero wind, HOR (dotted lines) and GM (symbols). 
(a)

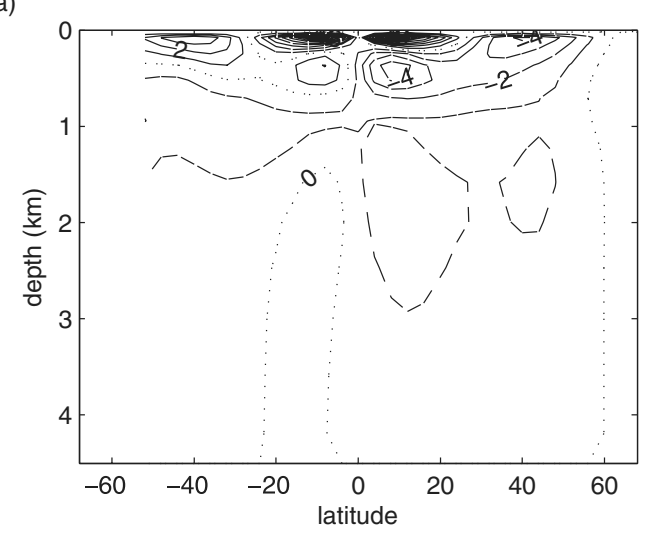

(b)

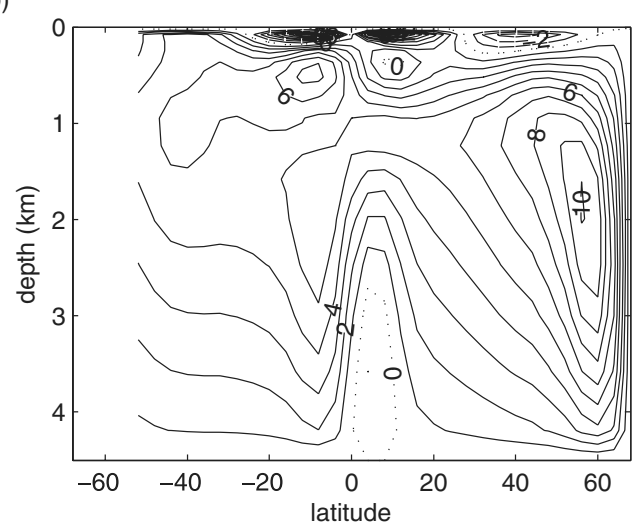

Fig. 13. Contour plots (interval $1 \mathrm{~Sv}$ ) of the (a) Pacific and (b) Atlantic meridional overturning for $K_{\mathrm{V}}=0.0053 \mathrm{~cm} \mathrm{~s}^{-1}$ in the $\mathrm{HOR}$ experiment.

their signature is no longer visible. This artefact is not seen in the HOR-NW experiment.

Additional to the differences in the scaling of the overturning strength, the sensitivity of the pycnocline depth also changes between HOR and GM. We address this point by investigating what processes determine the steady-state balance of density in the two regions where $\delta_{\rho}$ is calculated (Fig. 1). The effect of the deepening of the pycnocline with increasing $K_{\mathrm{V}}$ is visualized by presenting the results for four diffusivities $\left(K_{\mathrm{V}}=\right.$ $\left.0.53-3.0 \mathrm{~cm}^{2} \mathrm{~s}^{-1}\right)$.

The density balance in the upper $1500 \mathrm{~m}$ is very similar in each of the four cases shown in Figs 14 and 15. The surface balance is between horizontal (mainly meridional) advection and vertical advection induced by Ekman downwelling. Below about $500 \mathrm{~m}$ the contribution of horizontal advection decreases, which is accompanied by an increase in the density tendency due to lateral mixing. The latter is dominant between 1000 and 1500 $\mathrm{m}$. Within this interval the contribution of vertical advection decreases while the effect of vertical diffusion increases (both in absolute sense).

In the South Pacific (Fig. 14) vertical diffusion is the dominant contribution to the balance at depths below about $1500 \mathrm{~m}$. In both HOR and GM it is to leading order balanced by vertical advection in the depth range between 1700 and $3200 \mathrm{~m}$. Below $3200 \mathrm{~m}$, the tendency due to vertical advection decreases downward due to the no normal flow condition at the bottom. Zonal and meridional advection tend to compensate each other between 1500 and 3000 $\mathrm{m}$, but have a net effect below this depth interval (especially in HOR). It is interesting to note the similarity between the profiles of horizontal diffusion in HOR and the effect of eddy stirring in GM. In both experiments the effect of lateral mixing is small in the depth range where the advection-diffusion balance dominates, especially for the higher diffusivities.

In the North Atlantic the changes in density balance (Fig. 15) with vertical diffusivity are more difficult to interpret. Moreover, there is a clear difference between the results from the HOR and GM experiments. In the HOR experiment vertical diffusion is dominant below about $1700 \mathrm{~m}$. The contribution of vertical advection is either weakly compensating or of the same sign as vertical diffusion. The net negative tendency due to vertical advection and diffusion is balanced by both horizontal diffusion and net horizontal advection. Compared to HOR, the depth below which vertical diffusion dominates shifts downward in GM. Yet, in this case vertical advection is (weakly) compensating for all diffusivities. The dominant compensating contribution, however, is the mesoscale eddy flux, which is large at all depths below $1000 \mathrm{~m}$. The dominance of the eddy contribution can be understood from the fact that the deep western boundary current, and thus the eddy activity associated with it, is particularly intense close to the source of deep water. The effect of horizontal advection is difficult to assess, due to the change in sign with increasing diffusivity. At small diffusivities, where the sign is opposite to that in the HOR experiments, the contribution appears to be non-negligible.

\section{Discussion}

In this paper, we revisited the problem of the scaling behaviour of the global circulation versus vertical diffusivity. Previous studies (Wright and Stocker, 1992; Knutti et al., 2000; Dalan et al., 2005; Dijkstra, 2008) suggested that the scaling relations are different between the Pacific and Atlantic. Here, we tested the robustness of that result, using restoring conditions on density and an idealized model configuration. In particular, we evaluated if the scaling depends on (1) the presence of wind stress and (2) the choice between horizontal or GM mixing to represent the effect of mesoscale eddies. We found that for each of the four cases considered, there is a range of diffusivities where the scaling is different between the Pacific and Atlantic.

Our results indicate that the asymmetry in scaling behaviour is only manifest in the overturning rate and not in the pycnocline depth. This finding is in contrast with the results of Dijkstra (2008), who observed that the pycnocline depth in the Atlantic is less sensitive to changes in $K_{\mathrm{V}}$ than that in the Pacific. He 

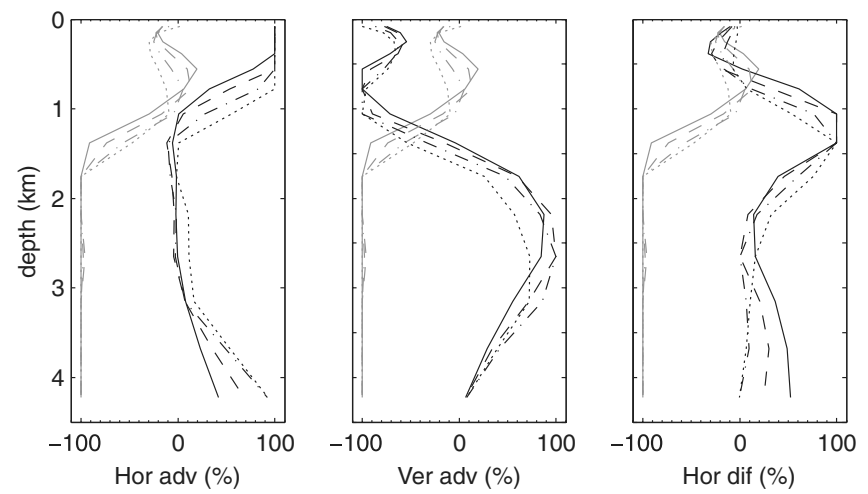

$3.0 \mathrm{~cm}^{2} \mathrm{~s}^{-1}$

(b)
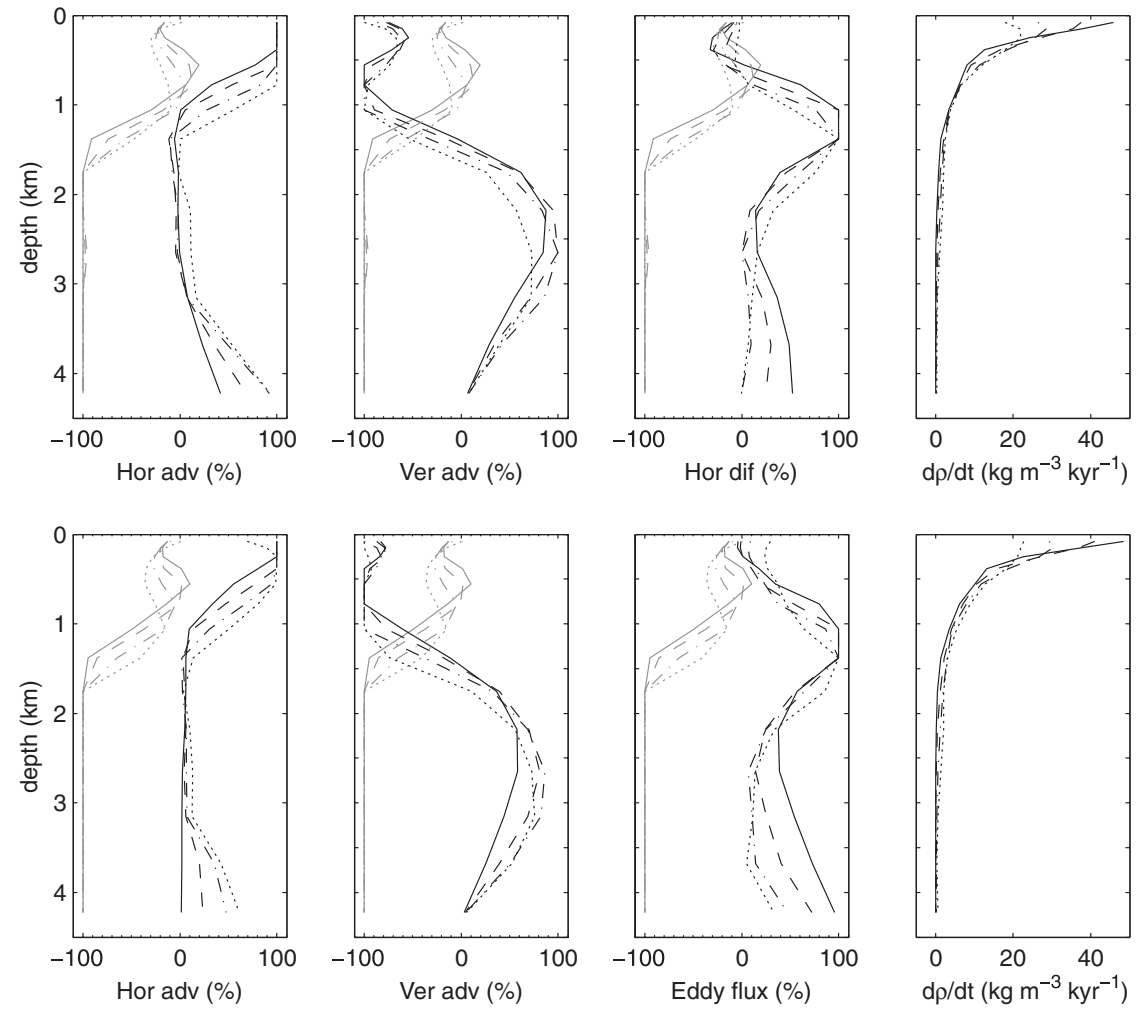

Fig. 14. Normalized density tendencies as function of depth for the centre of region P in (a) HOR and (b) GM. The line style indicates the value of the diffusivity. The contribution of vertical diffusion is shown in each of the first three panels by grey lines. From left to right the black lines represent the contributions of horizontal advection, vertical advection and (a) horizontal diffusion or (b) parametrized mesoscale eddies. Convection does not contribute to the balances in any of the results shown. The profiles are normalized at each depth by the contribution that is largest in absolute value (shown in rightmost panel). Since the total density tendency is negligible, the contributions indicated by the grey line and the three black lines sum to zero for a given depth and $K_{\mathrm{V}}$.

suggested that this difference is responsible for the asymmetry in the scaling of the MOC by arguing that the ratio of $\Psi$ and $\delta_{\rho}$, which is proportional to the characteristic velocity scale $V$ in eq. (1), has the same sensitivity in both basins. Instead, the results presented here imply that the asymmetry in scaling is due to the relation between overturning rate and pycnocline depth being different for the Pacific and Atlantic.

The mechanism proposed by Dijkstra (2008) assumes that the geostrophic relation $V \propto \Delta \rho \delta_{\rho}$ (eq. 1a) holds. This means that, if the scaling of $V$ is to be the same in both basins, while the scaling of $\delta_{\rho}$ differs, the typical density contrast $\Delta \rho$ is required to vary as function of $K_{\mathrm{V}}$. Although the use of restoring conditions does not prevent changes in the typical density contrast, we demonstrated for the 'no wind' case that the actual sensitivity of $\Delta \rho$ is rather weak. This implies that the mechanism of Dijkstra (2008) cannot operate in the experiments performed in this study. Furthermore, it should be noted that the results of Dijkstra (2008) were obtained using non-zero wind and horizontal tracer mixing, that is, the equivalent of the HOR case presented here, for which we showed that the asymmetry in the scaling of the overturning is for a large part related to the impact of spurious diapycnal mixing.

We have not been able to formulate an alternative theory that explains the difference in scaling between Pacific and Atlantic. Yet, our results are consistent with some characteristics of the simple pycnocline model of Gnanadesikan (1999): first, a single depth scale is sufficient to describe the global dynamics; and secondly, the effects of southern ocean winds and eddies are important for the scaling behaviour. However, the closure assumption used by Gnanadesikan (1999) for the North Atlantic Deep Water formation $\left(\Psi_{\mathrm{A}} \propto \delta_{\rho}^{2}\right)$ is not supported by our results from the experiments with non-zero wind (HOR and GM). At higher diffusivities the thermal wind relation also breaks down in the experiments without wind (HOR-NW and GM-NW).

Wright and Stocker (1992) suggested that a reduction in sensitivity to $K_{\mathrm{V}}$ of the Atlantic overturning compared to the predicted 2/3-dependence may be a simple consequence of the presence of deep convection, which represents an additional 


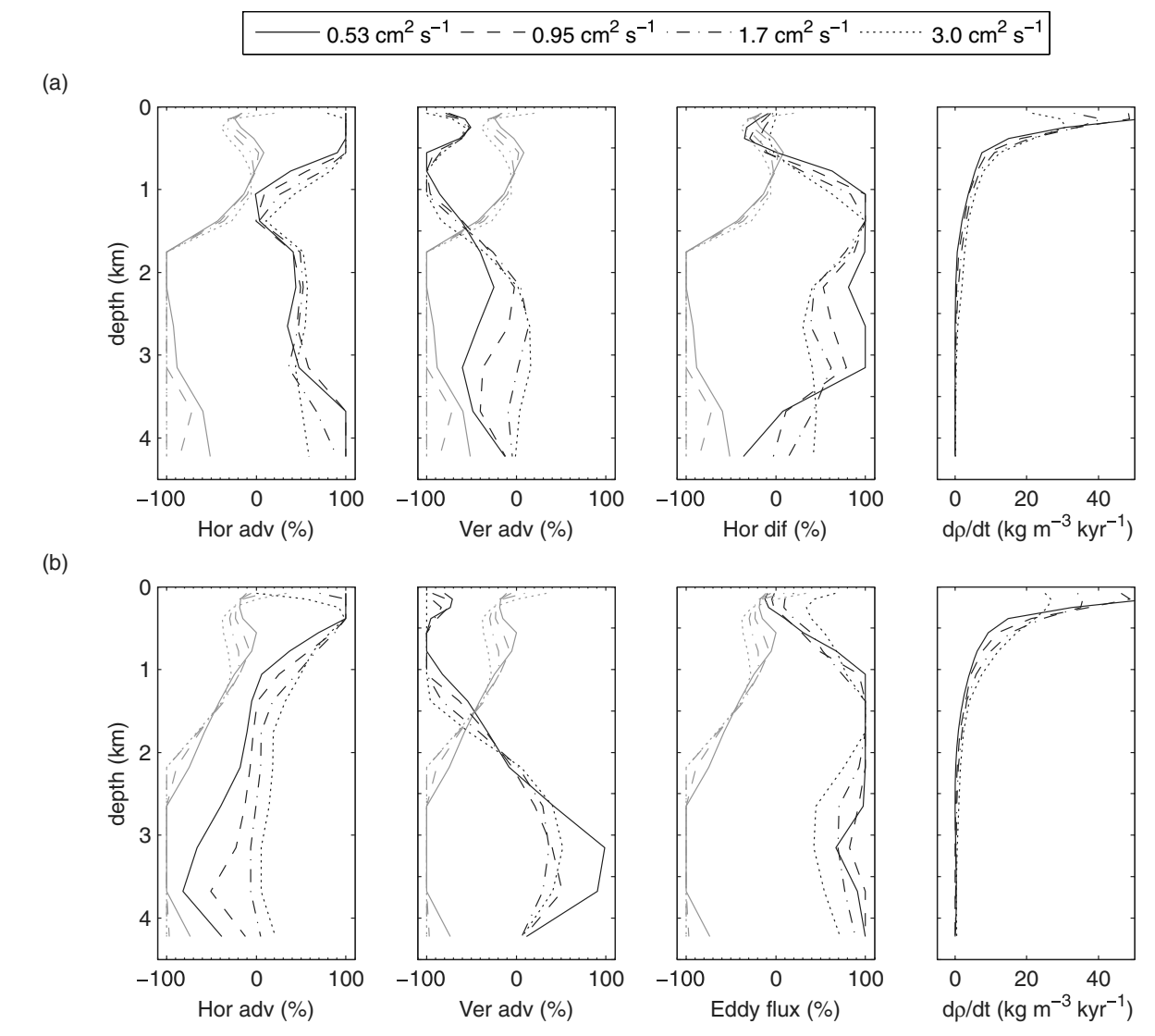

Fig. 15. Contributions to the density tendency as in Fig. 14, but now for the centre of region A.

vertical mixing process not included in the classical scaling theory. They also noted, though, that such reduced sensitivity does not occur when a single basin is considered, despite the vicinity of a convective region. We therefore emphasize that in the configuration under study the overturning strength either measures the net upwelling $\left(\Psi_{\mathrm{P}}\right)$ or the net downwelling $\left(\Psi_{\mathrm{A}}\right)$ in a basin, rather than both up- and downwelling as in a single basin.

We showed that, in all experiments, $\Psi_{\mathrm{P}}$ is accurately predicted by the scaling law that represents the vertical advection-diffusion balance. For the HOR and GM experiments we demonstrated that this result is compatible with the actual density balances. For these experiments we also showed that other processes than vertical advection and diffusion dominate the density balance in the Atlantic. Still, this only partly explains the departure from the advection-diffusion relation in the Atlantic, because $\Psi_{\mathrm{A}}$ measures downwelling, rather than upwelling. For the HOR-NW and GM-NW experiments it was noted that the fraction that upwells within the Atlantic varies with $K_{\mathrm{V}}$, as a result of changes in the meridional velocity structure at higher diffusivities. The changes coincide with the divergence of $\delta_{\rho}$ and the flow-based depth scale $\delta_{v}$, which for the GM-NW results was shown to be diagnostic of an increasing complexity of the zonal structure of the meridional velocity, which is not accounted for in classical scaling theory. So, the reduced sensitivity to $K_{\mathrm{V}}$ of the Atlantic overturning is probably not solely related to the presence of convection, as was put forward by Wright and Stocker (1992). Rather, we conclude that the asymmetry in scaling is the signature of a fundamental break down of the classical scaling laws.

The results from the HOR-NW and GM-NW experiments show that in absence of wind forcing the scaling is only weakly affected by the choice of the mixing scheme. This suggests that when the implicit model THCM (De Niet et al., 2007) is used to study the dynamics of buoyancy-driven flows, the results have validity beyond the scope of horizontal tracer mixing (to the use of which the model is presently limited). In presence of wind, however, the results become strongly influenced by the spurious cross-isopycnal mixing. This finding disagrees with the conclusion reached by Knutti et al. (2000) on the basis of their results with a model consisting of interconnected zonally averaged basins. The difference is partly explained by the absence of the Veronis (1975) effect in two-dimensional simulations. In addition to changes in the MOC scaling we found that the use of GM instead of horizontal diffusion results in a significant increase of the sensitivity of $\delta_{\rho}$ to $K_{\mathrm{V}}$. The analysis in Section 4.2 suggests that this can be attributed to changes in the processes that control the steady state balance of density in the Atlantic. 
In particular we note the reverse in sign of the contribution due to vertical advection.

Although the idealized two-basin formulation used here already captures several important characteristics of the real-world ocean, the scaling relations have been obtained using rather severe restrictions. To begin with, the properties of the overturning circulation considered in this work are strongly determined by the configuration. We expect the results would change if a realistic global geometry would be used, in which there is a considerable difference in relative area and latitudinal extent of the Atlantic and (Indo-)Pacific basins (Dalan et al., 2005). Our results provide no indication about the effect of prescribing a strongly asymmetric geometry, but it is more likely to enhance the asymmetry in scaling than to reduce it. Another natural extension of this study would be the use of more realistic boundary conditions for temperature and salinity. In reality these tracers interact with the atmosphere in a fundamentally different way, thus allowing the surface density field to be much stronger influenced by the flow than is possible with restoring boundary conditions. As suggested by Dalan et al. (2005), this may lead to different scaling behaviour. Furthermore, with the use of more realistic boundary conditions, it would be useful to adequately relate temperature and salinity to density through a non-linear equation of state, which would probably also affect the results. We finally note that, in reality, vertical mixing is a strongly inhomogeneous process, which is determined by mixing efficiency and energy constraints (Munk and Wunsch, 1998). By using a constant value of $K_{\mathrm{V}}$ these inhomogeneities are not adequately captured. Still, despite the relative simplicity of our model, we believe our configuration is able to provide new insights in the dynamics of the global overturning circulation, and is worth exploring further to obtain a more complete understanding of the results.

\section{Conclusion}

In summary, our results demonstrate that a difference between the Pacific and Atlantic in the scaling behaviour with vertical diffusivity is not a robust feature of multi-basin overturning circulations. In absence of wind and at sufficiently low diffusivity, the scaling is in accordance with the classical scaling relations in both basins, regardless of which alternative of the mesoscale eddy parametrization is employed. Only at higher diffusivities the results start to deviate from the classical relations in such a way that the scaling of the overturning strength becomes asymmetric. In presence of wind, the choice of the mixing scheme has a large impact on the results. With horizontal mixing, the scaling is different between the Pacific and Atlantic across the entire range of diffusivities under consideration, but this can to a large degree be attributed to the effect of false diapycnal fluxes. With GM the scaling is the same at lower values of $K_{\mathrm{V}}$, but with increasing diffusivity the sensitivity of the overturning strength in the Atlantic is reduced compared to that in the Pacific. The scaling of the pycnocline depth is similar in the two basins, irrespective of the absence or presence of wind forcing and of the choice of the lateral mixing scheme. In contrast to previous work (Dijkstra, 2008), the occurrence of asymmetric scaling can not be explained by a simple relation between overturning strength and pycnocline depth. The scaling behaviour of the overturning in a two-basin (global) ocean model is thus much more complicated than that of buoyancy-driven flows in a single hemispheric model (Park and Bryan, 2000). Therefore, a continued systematic investigation of the processes that determine the pycnocline depth, and of its relation to the overturning strength, is needed to obtain a more complete picture of the dynamics of the global overturning circulation.

\section{Acknowledgments}

All computations were done on the Huygens IBM p6 supercomputer at SARA Amsterdam. Use of these computing facilities was sponsored by the National Computing Facilities Foundation (N.C.F.) under the project SH084-08 with financial support from the Netherlands Organization for Scientific Research (N.W.O.). This work was further supported by a N.W.O. Toptalent Grant to one of the authors (MdT). The authors would like to thank both anonymous referees for their helpful comments on the manuscript.

\section{References}

Bryan, F. 1987. Parameter sensitivity of primitive equation ocean general circulation models. J. Phys. Oceanogr. 17, 970-985.

Bryan, K. and Cox, M. 1967. A numerical investigation of the oceanic general circulation. Tellus 19, 54-80.

Colin de Verdière, A. 1988. Buoyancy driven planetary flows. J. Mar. Res. 46, 215-265.

Dalan, F., Stone, P. H., Kamenkovich, I. V. and Scott, J. R. 2005. Sensitivity of the ocean's climate to diapycnal diffusivity in an EMIC. Part I: equilibrium state. J. Climate 18, 2460-2481.

De Niet, A., Wubs, F., Terwisscha van Scheltinga, A. and Dijkstra, H. 2007. A tailored solver for bifurcation analysis of ocean-climate models. J. Comp. Phys. 227, 654-679.

Dijkstra, H. A. 2005. Nonlinear Physical Oceanography: A Dynamical Systems Approach to the Large Scale Ocean Circulation and El Niño, 2nd Revised and Enlarged edition. Springer, New York, 532 pp.

Dijkstra, H. A. 2008. Scaling of the Atlantic meridional overturning circulation in a global ocean model. Tellus 60A, 749-760.

Gent, P. and McWilliams, J. 1990. Isopycnal mixing in ocean circulation models. J. Phys. Oceanogr. 20, 150-155.

Gerdes, R., Koberle, C. and Willebrand, J. 1991. The influence of numerical advection schemes on the results of ocean general circulation models. Clim. Dyn. 5, 211-226.

Gnanadesikan, A. 1999. A simple predictive model for the structure of the oceanic pycnocline. Sciences $\mathbf{2 8 3}, 2077-2079$.

Hu, D. 1996. On the sensitivity of thermocline depth and meridional heat transport to vertical diffusivity in OGCMs. J. Phys. Oceanogr. 26, 1480-1494. 
Klinger, B. A., Drijfhout, S., Marotzke, J. and Scott, J. R. 2003. Sensitivity of basinwide meridional overturning to diapycnal diffusion and remote wind forcing in an idealized atlantic-southern ocean geometry. J. Phys. Oceanogr. 33, 249-266.

Knutti, R., Stocker, T. F. and Wright, D. G. 2000. The effects of subgridscale parameterizations in a zonally averaged ocean model. J. Phys. Oceanogr. 30(11), 2738-2752.

Kuhlbrodt, T., Griesel, A., Montoya, M., Levermann, A., Hofmann, M. and co-authors. 2007. On the driving processes of the Atlantic meridional overturning circulation. Rev. Geophys. 45, RG2001.

Lazar, A., Madec, G. and Delecluse, P. 1999. The deep interior downwelling, the Veronis effect, and mesoscale tracer transport parameterizations in an OGCM. J. Phys. Oceanogr. 29, 2945-2961.

Ledwell, J. R., Watson, A. J. and Law, C. S. 1993. Evidence for slow mixing across the pycnocline from an open-ocean tracer-release experiment. Nature 364, 701-703.

Lineikin, P. 1955. On the determination of the thickness of the baroclinic layer in the sea. Dokl. Akad. Nauk USSR 101, 461-464.

Marotzke, J. 1997. Boundary mixing and the dynamics of threedimensional thermohaline circulations. J. Phys. Oceanogr. 27, 1713-1728.

Marotzke, J. and Willebrand, P. 1991. Multiple equilibria of the global thermohaline circulation. J. Phys. Oceanogr. 21, 1372-1385.

Munk, W. 1966. Abyssal recipes. Deep Sea Res. 13, 707-730.

Munk, W. and Wunsch, C. 1998. Abyssal recipes II: energetics of tidal and wind mixing. Deep Sea Res. 45, 1977-2010.

Pacanowski, R. and Griffies, S. 2000. MOM 3.0 Manual. Technical report, Geophysical Fluid Dynamics Laboratory/NOAA. Available online at http://www.gfdl.noaa.gov/.

Park, Y. and Bryan, K. 2000. Comparison of thermally driven circulations from a depth-coordinate model and an isopycnal-layer model.
Part I: scaling-law sensitivity to vertical diffusivity. J. Phys. Oceanogr. 30, 590-605.

Polzin, K. L., Toole, J. M., Ledwell, J. R. and Schmitt, R. W. 1997. Spatial variability of turbulent mixing in the abyssal ocean. Sciences 276, 93-96.

Redi, M. 1982. Oceanic isopycnal mixing by coordinate rotation. $J$. Phys. Oceanogr. 12, 1154-1158.

Robinson, A. and Stommel, H. 1959. The oceanic thermocline and the associated thermohaline circulation. Tellus 11, 295-308.

Toggweiler, J. and Samuels, B. 1998. On the ocean's large scale circulation in the limit of no vertical mixing. J. Phys. Oceanogr. 28, 1832-1852.

Toole, J. M., Polzin, K. L. and Schmitt, R. W. 1994. Estimates of diapycnal mixing in the abyssal ocean. Sciences 264, 1120-1123.

Vallis, G. K. 2000. Large-scale circulation and production of stratification: effects of wind, geometry, and diffusion. J. Phys. Oceanogr. 30, 933-954.

Veronis, G. 1975. The role of models in tracer studies. In: Numerical Models of Ocean Circulation. Natl. Acad. Sci., 133-146 pp.

Von der Heydt, A. and Dijkstra, H. A. 2007. Localization of multidecadal variability. Part I: cross-equatorial transport and interbasin exchange. J. Phys. Oceanogr. 37, 2401-2414.

Weaver, A. J. and Sarachik, E. S. 1990. On the importance of vertical resolution in certain ocean general circulation models. J. Phys. Oceanogr. 20, 600-609.

Welander, P. 1959. An advective model of the ocean thermocline. Tellus 11, 309-318.

Welander, P. 1971. The thermocline problem. Philos. Trans. Roy. Soc. Lond. 270A, 415-421.

Wright, D. G. and Stocker, T. F. 1992. Sensitivities of a zonally averaged global ocean circulation model. J. Geophys. Res. 97(C8), 12707-12730. 